\title{
Integrated modeling of ASDEX Upgrade plasmas combining core, pedestal and scrape-off layer physics
}

\author{
T. Luda ${ }^{\mathrm{a}, \mathrm{b}}$, C. Angioni ${ }^{\mathrm{a}}$, M. G. Dunne ${ }^{\mathrm{a}}$, E. Fable ${ }^{\mathrm{a}}$, A. \\ Kallenbach $^{\mathrm{a}}$, N. Bonanomi ${ }^{\mathrm{a}}$, P. A. Schneider ${ }^{\mathrm{a}}$, M. Siccinio ${ }^{\mathrm{a}}$, \\ G. Tardini ${ }^{\mathrm{a}}$, the ASDEX Upgrade Team ${ }^{\mathrm{c}}$, and the \\ EUROfusion MST1 Team ${ }^{\mathrm{d}}$ \\ a) Max-Planck-Institut für Plasmaphysik, Boltzmannstrasse 2, D-85748 \\ Garching, Germany \\ b) Aix-Marseille Université, CNRS, PIIM UMR 7345, 13397 Marseille, France \\ c) see the author list of H. Meyer et al. 2019 Nucl. Fusion 59112014 \\ d) see the author list of B. Labit et al. 2019 Nucl. Fusion 590860020 \\ E-mail: teobaldo.luda.di.cortemiglia@ipp.mpg.de
}

\begin{abstract}
The design of future fusion reactors and their operational scenarios requires an accurate prediction of the plasma confinement. We have developed a new model that integrates different elements describing the main physics phenomena which determine plasma confinement. In particular, we are coupling a new pedestal transport model, based on empirical observations, to the ASTRA transport code, which, together with the TGLF turbulent transport model and the NCLASS neoclassical transport model, allows us to describe transport from the magnetic axis to the separatrix. We also coupled a simple scrape-off layer model to ASTRA, which provides the boundary conditions at the separatrix, which are a function of the main engineering parameters. By this way no experimental data of the kinetic profiles is needed, and the only inputs of the model are the magnetic field, the plasma current, the heating power, the fueling rate, the seeding rate, the plasma boundary, and the effective charge. In the modeling work-flow, first a scan in pedestal pressure is performed, by changing the pedestal width. Then the pedestal top pressure is determined using the MISHKA MHD stability code. This modeling framework is tested by simulating ASDEX Upgrade discharges. We show comparisons with experimental fueling, power, and current scans. The changes of the pedestal structure and the gradients in the plasma core, due to the different combinations of fueling, heating power, and plasma current, are well captured by the model. We also show that the predicted pedestal and global stored energies are in good agreement with the experimental measurements, and more accurate with respect to the prediction of the IPB98 $(y, 2)$ scaling law. The long term goal is to obtain a robust and complete model which can be used to identify important hidden dependencies affecting global plasma confinement, which are difficult to capture by statistical regressions on global parameters.
\end{abstract}


Integrated modeling of ASDEX Upgrade plasmas combining core, pedestal and scrape-off layer physics 2

\section{Introduction}

The design of future fusion reactors and the definition of the scenarios of operation require an accurate prediction of the plasma confinement and of the plasma density and temperature profiles, as these determine the fusion performance and thereby also the production of net electric energy.

Empirical approaches can be used [1-3], mostly based on statistical analyses performed on large databases of experimental data, and these usually provide scaling laws of the plasma stored energy (or, equivalently, of the energy confinement time) commonly in the form of multi-variate log-linear regressions [4-6]. These scaling laws for the global plasma confinement are certainly very valuable and useful tools for zero dimensional (0D) predictions, and even in providing a scaling factor in (semiempirical modeling for the prediction of the radial kinetic profiles. They translate the complexity of the entire physical system of the plasma into a relatively simple log-linear regression based on reduced essential sets of engineering parameters, most commonly the plasma size, the plasma current, the magnetic field, the plasma density and the plasma composition. The large experience with the operation of tokamaks has shown that these scaling laws can robustly capture some dominant dependencies on critical engineering parameters, for instance that on the plasma current and to some extent on the plasma size, but at the same time has revealed that some of the other dependencies, like those on the density and the heating power, are connected to a complex combination of various physical effects which depend on several other variables and can be hardly described within a zero-dimensional approach for all domains of operation [7-9]. Moreover, since these statistical approaches do not contain any (or little) element of the physics which is behind these dependencies, their predictive reliability has to be considered with care, as the extrapolation to reactor conditions requires to move largely outside of the multi-dimensional domain of data over which the statistical analysis has been performed.

In parallel, the prediction of the plasma kinetic profiles, including their dynamical evolution, is usually obtained by means of one dimensional (1D) approaches which combine different modules describing plasma transport and sources at various levels of integration. This can imply the coupling of different modules to describe various transport mechanisms and sources [10-19], while adopting boundary conditions from measurements inside the confined plasma, but also the connection between core and edge, including a description of the pedestal [20-29]. Today these integrated modeling workflows can rely on increasingly sophisticated theory-based models for turbulent transport, particularly applicable in the core of the confined plasma [3033]. Here the completeness and realism of the physics description is naturally limited by requirements in computational time, which is particularly severe when simulating entire discharges. Moreover, since at least in this context no single model for plasma transport exists which can be applied over the entire plasma cross section, predictions unavoidably rely on a set of empirical elements, which usually consist in the definition of boundary conditions which are taken from experimental measurements outside the radial simulation domain. Obviously the use of experimental measurements as boundary conditions strongly limits the possibilities of this approach to the prediction of non-existing devices. This limitation is particularly severe in the $\mathrm{H}$-mode confinement regime and the related operational scenarios which are foreseen for ITER [34], and which could be also considered for a future fusion reactor, since in this regime the physics regulating the different plasma regions becomes even more complicated to describe. The $\mathrm{H}$-mode confinement regime is characterized by the 
development of an external region of the plasma, usually called pedestal, in which radial transport is strongly reduced, compatible with the establishment of very strong radial gradients of both density and temperature profiles, leading to a strong increase of the plasma stored energy. These strong gradients are usually limited by the occurrence of edge localized modes (ELMs), and the trigger of these instabilities turns out to be sufficiently well described by magneto-hydrodynamic (MHD) models based on linear physics. This, combined with particle and energy transport levels, result in a determination of the pedestal height and width. The linear stability of these modes depends on the parameters of the core, particularly through the plasma pressure and the consequent impact on the Shafranov shift of the magnetic equilibrium, while, at the same time, the core profiles depend on the boundary conditions set by the pedestal stability. Such a strong coupling clearly requires a modeling framework which describes both core and edge self-consistently [27-29]. The pedestal stability is also affected by the actual shape of the pressure profiles, which are influenced by the radial transport and also by the pressure values at the separatrix, directly connecting pedestal stability with the properties of the scrape-off layer (SOL). In tokamak operation, and particularly in a reactor, SOL parameters have to respect the constraints imposed by power exhaust and thereby an interesting and highly relevant problem becomes the study and the prediction of how the plasma confinement is affected by the SOL and the divertor conditions. This also implies that a complete model for plasma confinement cannot exclude the SOL, which is usually modeled by highly sophisticated and computationally expensive 2D or 3D edge codes, which however are not practical to be coupled with a $1 \mathrm{D}$ transport solver.

The ultimate goal would be to develop a complete and general integrated modeling tool which combines highly realistic modules for the description of different plasma regions and in which the amount of empirical elements is kept to a minimum, and which uses as inputs only the plasma engineering parameters and the external actuators. It can be expected that these modeling tools would have the possibility of correctly predicting critical dependencies on various hidden parameters which cannot be captured by $0 \mathrm{D}$ scaling laws, providing more reliable predictions of the plasma confinement also for future devices.

We developed a new model, within the highly flexible modeling framework enabled by the ASTRA transport code [35], based on a workflow which allows the description of the coupling between SOL, pedestal and core, with a self-consistent treatment of the boundary conditions. The workflow combines theory-based components (like the quasi-linear TGLF [30] model for core turbulent transport, the NCLASS [36] model for neoclassical transport, the MHD code MISHKA [37] for the pedestal stability and the two-point model for the SOL $[38,39]$ ) with semi-empirical elements, in particular for the description of the transport in the pedestal region and for the connection between divertor and SOL parameters. We show that we can correctly reproduce some critical dependencies in agreement with the experimental trends, also in connection with the properties of the SOL, like for instance the impact of gas-puff on pedestal stability and confinement, which are outside the possibilities of usual scaling laws and which, to our knowledge, have never been reproduced within an integrated modeling approach.

Section 2 describes in details all the physics based and empirical elements constituting the modeling workflow, with an explanation on the integration of the different components. Section 3 presents the ASDEX Upgrade (AUG) experimental cases taken into account for a first test of the model, illustrating the different combinations of plasma parameters considered. The results derived from this application are given in section 4, containing different subsections for each of the 
experimental scans studied, where we compare the prediction of the model with scaling laws and experimental data. Summary and conclusions are presented in section 5 .

\section{Description of the integrated modeling workflow}

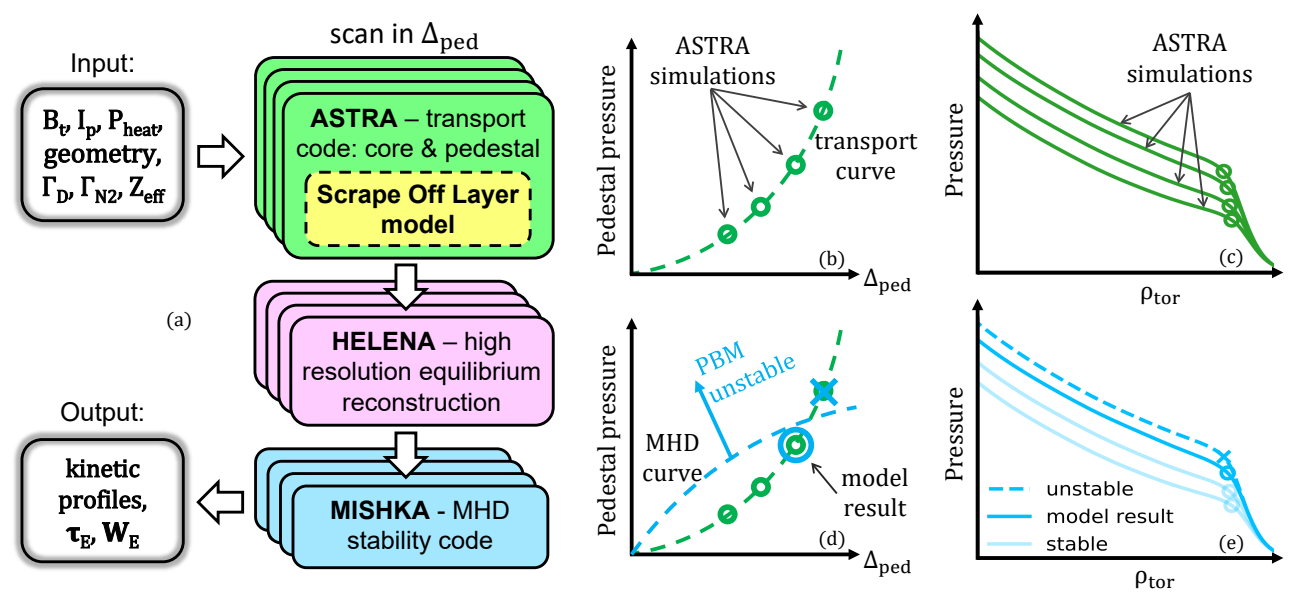

Figure 1. Chart representation of the modeling workflow (a). Multiple parallel ASTRA simulations calculate the kinetic profiles for different values of the pedestal width (c). The pedestal model integrated in ASTRA gives a transport constraint which determine the pedestal pressure for a given pedestal width (b). MISHKA tests the stability of the resulting profiles to find the highest stable pedestal pressure $(\mathrm{d}, \mathrm{e})$.

This section provides a detailed description of the modeling framework and of the transport models which are applied in the different regions of the plasma. We developed a tokamak plasma simulation tool which takes as inputs only global plasma parameters like the magnetic field, the plasma current, the deuterium fueling rate, the nitrogen seeding rate, the effective charge, as well as the auxiliary heating powers and the plasma boundary, and is capable of predicting the radial profiles of temperatures and densities until the last closed flux surface (LCFS) and, consequently, also the total stored energy and the confinement time.

Figure 1 (a) depicts the modeling workflow, which is based on the approach of performing multiple simulations in parallel, represented by the multiple ASTRA blocks in the workflow. In each of these multiple simulations, a different value of the pedestal width $\Delta_{\text {ped }}$ is assumed, in order to scan the pedestal pressure. A scrape-off layer (SOL) model has also to be included, since also the boundary conditions for the temperature and density profiles at the LCFS are predicted in the simulations. All of these transport components are simultaneously included in the simulations, which therefore provide predictions of the entire plasma radial profiles. However, particularly in H-mode, the different regions of the plasma, the core, the pedestal and the SOL, require different models that have to be combined in the integrated modeling framework. In this section we first provide a general description of the modeling workflow, introducing the various numerical tools which are included and how these are applied to obtain the final prediction of the plasma profiles from the magnetic axis to the LCFS. Subsection 2.1 illustrates all the elements constituting the ASTRA 
transport simulations, and is divided into four parts describing: how the SOL model sets the boundary conditions of the simulation in subsection 2.1.1, how transport is modeled in the pedestal in subsection 2.1.2 and in the core in subsection 2.1.3, and finally the methodology which is applied for the integration of the different transport components in the different plasma regions in subsection 2.1.4. Subsection 2.2 provides information on the calculations of the MHD stability of the pedestals predicted in each one of the multiple ASTRA simulations. Subsection 2.3 summarizes the assumptions introduced into the model and discusses their applicability to other experimental conditions and the sensitivity of the model result to the values of the free parameters assumed in the model.

Tokamak plasmas in the H-mode confinement regime are characterized by two distinct plasma regions: the core, distinguished by stiff profiles due to turbulent transport, and the pedestal, a peripheral region of the plasma where turbulence is strongly suppressed. This edge transport barrier allows the development of strong gradients, which dramatically increase the stored energy. Plasma confinement is therefore strongly related to the pedestal height, or the pedestal pressure. The pedestal height is defined by two quantities: the pedestal width and the pedestal gradient, which is regulated by transport and sources. While many different models can describe core transport, no theory based numerical model exist to provide a robust determination of transport in the pedestal region. To solve this problem we developed a new pedestal transport model based on empirical observations. In figure 2(a) of Ref. [40] it is shown that the pedestals of different machines all exhibit a similar feature: a constant ratio between the averaged pedestal electron temperature gradient (in real space units) and the pedestal top temperature $<\nabla T_{\mathrm{e}}>/ T_{\mathrm{e} \text {,ped }} \approx$ const $=0.5$. This condition has been implemented in ASTRA, so that by fixing a value of the pedestal width, the electron heat conductivity $\chi_{\mathrm{e}, \mathrm{ped}}$ that fulfills this condition is found. We then consider the ion heat conductivity $\chi_{\mathrm{i}, \text { ped }}$ and the particle diffusivity $D_{\mathrm{n} \text {,ped }}$ as given by the sum of neoclassical transport (calculated with NCLASS [36]) and terms proportional to $\chi_{\mathrm{e}, \mathrm{ped}}$, as described in Sec. 2.1.2. The application of this empirical condition results in a pedestal transport constraint, which gives a relation between the pedestal width and height. Thereby, for a certain value of the pedestal width, we obtain the pedestal height and the pedestal gradient.

With such a transport constraint the pedestal pressure increases with increasing width, as sketched in figure 1 (b), until the MHD stability limit is reached, as in figure 1 (d). The way the pedestal transport model is implemented in ASTRA, as discussed later on, requires to reach stationary conditions of the kinetic profiles for a given value of the pedestal width. Therefore many different ASTRA simulations with different values of the pedestal width need to be run in parallel in order to obtain a scan of the pedestal pressure. Figure 1 (c) shows the pressure profiles calculated by ASTRA (from the magnetic axis to the separatrix, although here shown only from $\rho_{\text {tor }}=0.5$ to better highlight the pedestal) for different values of the pedestal width. The MISHKA [37] MHD stability code is then run on each ASTRA simulation results to find the highest pedestal pressure that is stable to peeling-ballooning modes (PBM), corresponding to pre-ELM conditions. Figure 1 (e) shows the pressure profile which is the final result of the modeling workflow, from which we can calculate the energy confinement time and the stored energy.

The SOL model consists of a set of analytical formulas we integrated in ASTRA which set the boundary conditions (at the separatrix) for the transport simulation. To compute the heat and particle sources, and the non-inductive current drive ASTRA includes TORBEAM [41], and NBI and neutrals modules. The TORBEAM and NBI 
modules require as input all the engineering parameters of the heating systems (e.g. power, angles, voltage of the beams, frequency of the gyrotrons, etc.) and can predict the power distribution. The radiated power is calculated by assuming a constant tungsten concentration $c_{\mathrm{W}}=2 \times 10^{-5}$ (arbitrarily chosen), which gives values of radiated power in the confined plasma comparable to the ones obtained from the measurements for the experimental cases considered in this work. This assumption can be substituted in the future with theory based impurity transport modeling, as part of future work. To calculate the ions densities we simply impose quasineutrality assuming boron as light impurity and that $Z_{\mathrm{eff}}$ is constant over the plasma radius. The SPIDER [42] equilibrium code is also coupled to ASTRA to calculate the plasma equilibrium with a prescribed plasma boundary. To calculate core turbulent and neoclassical transport the TGLF [30] quasilinear transport model and the NCLASS [36] model are used respectively.

With the inclusion of the new pedestal transport model, ASTRA provides a complete description of the transport coefficients in the whole radial domain of the confined plasma, allowing us to simulate the kinetic profiles, namely the electron and ion temperature and density $\left(T_{\mathrm{e}}, T_{\mathrm{i}}, n_{\mathrm{e}}, n_{\mathrm{i}}\right)$, and the current density $(j)$ profiles from the magnetic axis to the separatrix. The inputs of the model are the magnetic field, the total plasma current, the engineering parameters determining the auxiliary heating powers, the deuterium fueling rate, the nitrogen seeding rate, the plasma boundary, and the effective charge $Z_{\text {eff }}$.

\subsection{Transport simulations}

2.1.1. Scrape-off layer model A simple SOL model, consisting of a set of analytical formulas, has been included in ASTRA to provide the boundary conditions of the neutral density, and of the electron temperature and electron density at the last closed flux surface. We use the formulas for the separatrix electron temperature $T_{\mathrm{e}, \mathrm{sep}}$ and density $n_{\mathrm{e}, \mathrm{sep}}$ from Ref. [43] (equations 5 and 8 respectively) obtained through an extension of the 2-point model [38, 39]. For the boundary condition of the ion temperature we simply assume $T_{\mathrm{i} \text {,sep }}=2 T_{\mathrm{e}, \mathrm{sep}}$. This is regularly observed in AUG experiments, and can be justified by the fact that the parallel heat conductivity in the SOL is smaller for the ions than for the electrons. This assumption might not be valid for detached divertor conditions, where one would expect that the difference between $T_{\mathrm{i}, \text { sep }}$ and $T_{\mathrm{e} \text {,sep }}$ becomes smaller. The experimental cases considered so far are all in attached divertor conditions. In order to take into account cases in fully detached conditions this assumption will have to be replaced with a more appropriate one, this will be done as part of future work. The $n_{\mathrm{e}, \mathrm{sep}}$ formula

$$
\begin{aligned}
& n_{\mathrm{e}, \mathrm{sep}}=0.35 \frac{2}{e}\left(\frac{2 \kappa_{0} \kappa_{\mathrm{z}}}{7 \pi q_{\mathrm{cyl}}}\right)^{2 / 7}\left(\frac{m_{\mathrm{D}}}{2}\right)^{0.5} R^{-0.5}\left(\frac{P_{\mathrm{sep}} B}{3 \pi<\lambda_{\mathrm{q}, \mathrm{HD}}><B_{\mathrm{p}}>}\right)^{3 / 14} \\
& (\gamma \sin (\alpha))^{-0.5}\left(1.510^{23} \mathrm{~Pa} /\left(\mathrm{at} \mathrm{m}^{-2} \mathrm{~s}^{-1}\right)\right)^{0.5} \mathrm{p}_{0}^{1 / 4}
\end{aligned}
$$

couples the plasma parameters in the divertor and midplane (at the separatrix), by assuming pressure balance. The formula also assumes that momentum losses, power losses, and divertor heat flux broadening can be combined into a coefficient, which is found by regression analysis on AUG to depend mainly on the divertor neutral pressure. For fully detached divertor conditions, which are relevant for future fusion reactors (e.g. DEMO), the hypothesis of pressure balance would not hold anymore due to significant momentum losses, so the formula should be modified to include also convective flows (as done in Ref. [44]), which become important at low temperatures 
(i.e. detached conditions) [45]. The midplane parameters required by the formula are the power crossing the separatrix $P_{\text {sep }}$, the major radius, the main ion mass, the magnetic field and the safety factor (also used to calculate $\lambda_{\mathrm{q}}[46,47]$ ), and are calculated by ASTRA. For the divertor parameters we assume constant (not changed among the different cases considered in this work) the sheath energy transmission factor $\gamma=6$, the impact angle of the field line at the outer target $\alpha=3.3^{\circ}$, and $Z_{\text {eff,div }}=1.3$ (used to calculate $\kappa_{\mathrm{z}}$ ), values of which are typical for AUG [48]. The only remaining unknown parameter is the divertor neutral pressure $p_{0}$, which is the main term of the formula. To estimate it a scaling has been derived using AUG data, obtained with a baratron [49] in the configuration with the divertor DivIII [50]. The regression has been performed on 116 data-points, using as variables the deuterium fueling rate $\Gamma_{\mathrm{D}}\left[10^{19} \mathrm{e} / \mathrm{s}\right]$, the nitrogen seeding rate $\Gamma_{\mathrm{N} 2}\left[10^{19} \mathrm{e} / \mathrm{s}\right]$, the NBI power $P_{\mathrm{NBI}}[\mathrm{MW}$ (which represents the fueling provided by the NBI), and (because AUG operates with a cryopump) the pumping speed expressed in relative velocity $v_{\text {pump }}$ [\%] ( 1 if operating on liquid helium, 0.5 if on liquid nitrogen, 0.2 if turned off). The result of this regression reads,

$$
p_{0}=0.174 \Gamma_{\mathrm{D}}^{0.63} \Gamma_{\mathrm{N} 2}^{-0.057} P_{\mathrm{NBI}}^{0.33} v_{\text {pump }}^{-0.67} .
$$

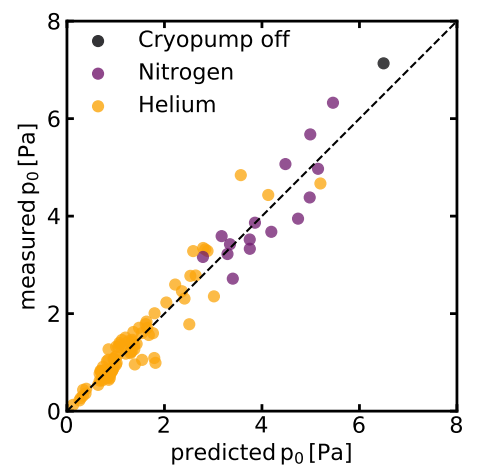

Figure 2. Comparison between the predicted and measured divertor neutral pressure. The color code shows the operation of the cryopump with liquid helium (orange), liquid nitrogen (purple), or turned off (black).

Figure 2 shows the measured divertor neutral pressure compared to the one predicted. Remarkably, the coefficient of determination $R^{2}=0.948$ and the root mean squared error $R M S E=16.8 \%$ show the good description of the data by the scaling, allowing a robust evaluation of $n_{\mathrm{e}, \mathrm{sep}}$. We performed a log-linear regression instead of a linear regression since it provided a better result. For the cases without nitrogen seeding or NBI power the terms $\Gamma_{\mathrm{N} 2}$ or $P_{\mathrm{NBI}}$ are simply dropped out of the formula to avoid getting a null value of $p_{0}$. A histogram showing the distribution of the data used to compute the scaling is depicted in figure 3 . The range of parameters used for the scaling is wide enough to cover the AUG operational space, therefore we expect that it can be robustly applied to predict $p_{0}$ for AUG.

Due to the location of the baratron (figure 3 in Ref. [49]), the measurement of the divertor neutral pressure is influenced by the high-field-side high-density (HFSHD) front (observed in AUG and JET) [51, 52], a region of the HFS SOL where the density of the plasma reaches high values, approximately one order of magnitude higher then at 
Integrated modeling of ASDEX Upgrade plasmas combining core, pedestal and scrape-off layer physics8
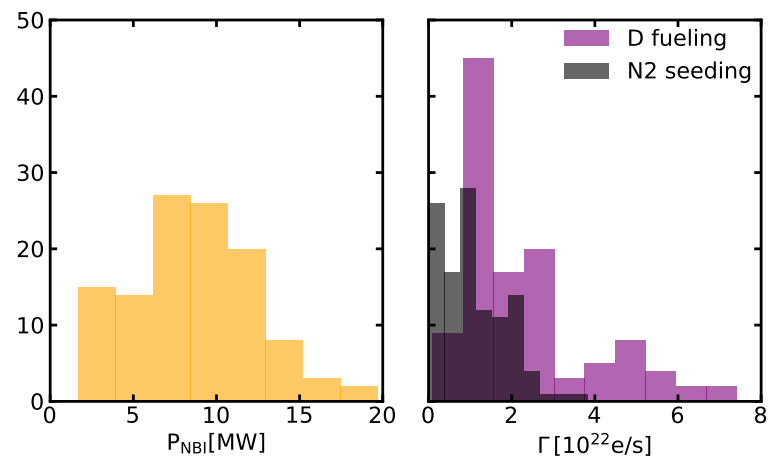

Figure 3. Histograms showing the number of observations in the database used for the regression as a function of the NBI power (left), and deuterium fueling (purple) and nitrogen seeding (black) rates (right). The histograms with deuterium fueling and nitrogen seeding are plotted with transparent colors, the darker region results from their overlap.

the separatrix. This high density front appears when operating with sufficient levels of gas fueling and heating power. The density of the front is reduced by impurity seeding since this increases the radiation, causing a decrease of the power exhausted by the confined plasma before reaching the HFS SOL and ionizing the particles associated with the HFSHD. The heating power also has a similar effect, if this is reduced the density of the HFS front decreases. Because of the influence of the HFSHD front on the measurement of divertor neutral pressure, we can include in the scaling the effect of nitrogen seeding (the main impurity used in AUG), and ultimately its effect on the separatrix density. Interestingly when performing the regression for the scaling the only type of heating source that seem to have an impact on the divertor neutral pressure is the NBI, while ECRH and ICRH seem to have no correlation. This could be due to the fact that the NBI is not only heating the plasma but it also provides a source of particle, although this should be rather small. Another cause could be related to a different increase of the radiated power caused by the different heating sources, but this has not been investigated in this work and could be analyzed in the future.

The source of neutrals crossing the separatrix is an important boundary condition since together with transport it defines the density profile in the pedestal region. The main source of neutrals is given by the wall gas inventory which is sustained by recycling and gas puffing, which we compute as

$$
\Gamma_{0, \text { wall }}=f_{\mathrm{R}} \Gamma_{\mathrm{e}, \mathrm{sep}}+c_{\mathrm{div}, \text { wall }}\left(\Gamma_{\mathrm{D}}-\Gamma_{\mathrm{pump}}\right) .
$$

The term $f_{\mathrm{R}} \Gamma_{\mathrm{e}, \text { sep }}$ represents recycling, where $\Gamma_{\mathrm{e}, \mathrm{sep}}$ is the electron flux leaving the plasma, and $f_{\mathrm{R}}$ is the recycled fraction. The term $c_{\mathrm{div}, \text { wall }}\left(\Gamma_{\mathrm{D}}-\Gamma_{\text {pump }}\right)$ represents the fueling given by gas puff valves $\Gamma_{\mathrm{D}}$ minus the cryopump absorption $\Gamma_{\text {pump }}$, considering that only a fraction $c_{\text {div,wall }}$ diffuses from the valves (in the divertor region) to the SOL (or to the wall). We can then estimate the source of neutrals crossing the LCFS as

$$
n_{0, \mathrm{sep}}=\alpha_{\mathrm{wall}, \mathrm{sep}} \frac{\Gamma_{0, \text { wall }}}{\nu_{0} A_{\mathrm{eff}}}=\alpha_{\text {wall }, \mathrm{sep}} \frac{\left(f_{\mathrm{R}} \Gamma_{\mathrm{e}, \mathrm{sep}}+c_{\mathrm{div}, \mathrm{wall}}\left(\Gamma_{\mathrm{D}}-\Gamma_{\mathrm{pump}}\right)\right)}{\nu_{0} A_{\mathrm{eff}}}
$$


Integrated modeling of ASDEX Upgrade plasmas combining core, pedestal and scrape-off layer physics9

ASTRA requires the neutral density at the separatrix $n_{0, \text { sep }}$ as input, which can be obtained dividing the neutrals flux $\Gamma_{0}$ by the neutrals velocity $\nu_{0}$ and the surface crossed by the neutrals $A_{\text {eff }}$. We assume that the most of the neutrals come from the divertor region and the wall at the outer midplane, which roughly correspond to one third of the LCFS $A_{\text {eff }}=A_{\mathrm{LCFS}} / 3$. The term $\alpha_{\text {wall,sep }}$ is a parameterization of the decay of the neutrals density caused by the ionization and charge exchange (CX) processes that occur during their flow from the wall to the confined plasma. The formula used to estimate this parameter has been obtained by fitting a database consisting of $\sim 1000$ values of $\alpha_{\text {wall,sep }}$ calculated for different values of the electron temperature and density, and of the distance between the LCFS and the wall. The calculation of $\alpha_{\text {wall,sep }}$ has been performed using the rate coefficients for ionization and CX of deuterium as a function of $T_{\mathrm{e}}$ as in [53], assuming a fixed decay length of the plasma density and temperature profiles in the SOL, and considering neutrals with a constant temperature of $T_{0}=5 \mathrm{eV}$, representing reflection of sheath accelerated ions and Franck-Condon neutrals

$$
\frac{\partial n_{0}}{\partial x}=-R_{\text {ion }} n_{\mathrm{e}} n_{0} / \nu_{0}-R_{\mathrm{CX}} n_{\mathrm{i}} n_{0} / \nu_{0}
$$

where $x$ is the radial coordinate. We solve this equation numerically, considering for simplicity $n_{\mathrm{e}}=n_{\mathrm{i}}$ and $T_{\mathrm{e}}=T_{\mathrm{i}}$ since the CX rate coefficient $R_{\mathrm{CX}}$ is not very sensitive to the temperature. We ignore line radiation and recombination since they are negligible in the range of temperatures we are considering. We then obtain $\alpha_{\text {wall,sep }}$ for different values of $n_{\mathrm{e}, \mathrm{sep}}, T_{\mathrm{e}, \mathrm{sep}}$, and distance between the wall and the LCFS $d_{\text {wall,sep. The }}$ scaling gives then, with a root mean squared error $R M S E<0.1 \%$ :

$$
\alpha_{\text {wall,sep }}=87.6-18.9 n_{\mathrm{e}, \mathrm{sep}}\left[10^{19} / \mathrm{m}^{3}\right]^{0.016}-67.2 T_{\mathrm{e}, \mathrm{sep}}[\mathrm{eV}]^{0.0027}-1.284 d_{\mathrm{wall}, \mathrm{sep}}[\mathrm{m}]^{0.939}(6)
$$

We then have an incoming source of neutrals with $T_{0}=5 \mathrm{eV}$. The energy of the neutrals does not change the result of the simulation since we estimate the incoming neutral particle flux $\Gamma_{0 \text {,sep }}$, and the neutral density depends then on the temperature since $\nu_{0} \propto \sqrt{T_{0} / m_{0}}$. So more energetic neutrals penetrate deeper into the confined plasma, but their density will be lower, and overall the effect will be balanced. We performed a sensitivity study of the effect of neutrals temperature $\left(T_{0}=5,3,2 \mathrm{eV}\right)$ on the density profile in the pedestal, but the density profile and its gradient were the same in all cases.

Another interesting aspect is that, for the different experimental cases considered (see Sec.3), even variations in the fueling rate by more than one order of magnitude produces only a small change in the neutrals density at the separatrix (i.e. an increase in the fueling rate of a factor 10 causes an increase in the neutrals density of less then a factor 2). This is consistent with the observations from Ref. [54], where the separatrix neutrals density appears to be in the order of $10^{16} / \mathrm{m}^{3}$ for all the different cases analyzed. This can be explained by the fact that when the fueling rate is increased, the separatrix density increases, causing a stronger decay of the neutrals density due to ionization and charge exchange, which partially compensate the stronger source of neutrals.

This simple SOL model allows us to capture the main effects of fueling on the boundary conditions of the ASTRA transport simulation, which can affect the pedestal stability and the plasma confinement.

2.1.2. Pedestal transport As previously described we make use of the experimental observation $\left\langle\nabla T_{\mathrm{e}}>/ T_{\mathrm{e} \text {,ped }} \approx\right.$ const $=0.5$ to calculate the transport coefficients 
in the pedestal region. This condition has been implemented in ASTRA, so that for a given pedestal width the electron heat conductivity $\chi_{\mathrm{e}, \text { ped }}$ is changed to fulfill this imposed condition. This is done by minimizing the difference between the value of $<\nabla T_{\mathrm{e}}>/ T_{\mathrm{e} \text {,ped }}$ calculated by ASTRA and the target value, modifying the absolute value of $\chi_{\mathrm{e}, \text { ped }}$ in a feedback iteration while evolving the kinetic profiles. The final value of $<\nabla T_{\mathrm{e}}>/ T_{\mathrm{e} \text {,ped }}$ matches the imposed condition with an error $<1 \%$. As previously described, many different ASTRA simulations are run in parallel to perform a scan (with discrete values) in pedestal width, where each simulation calculates the kinetic profiles for a different value of the pedestal width. We assume equal values of the pedestal widths for the electron and ion temperature and density $\Delta_{T_{e}}=\Delta_{T_{i}}=\Delta_{n_{e}}$. For each of these ASTRA simulations the kinetic profiles are evolved until the condition $<\nabla T_{\mathrm{e}}>/ T_{\mathrm{e} \text {,ped }}=0.5$ is satisfied and stationary conditions are reached. We then define the ion heat diffusion coefficient as $\chi_{\mathrm{i} \text {,ped }}=\chi_{\mathrm{e} \text {,ped }}+\chi_{\mathrm{i} \text {,neo }}$, where $\chi_{\mathrm{i} \text {,neo }}$ is the neoclassical ion heat diffusivity, calculated with NCLASS, while the neoclassical electron heat diffusivity $\chi_{\mathrm{e}, \text { neo }}$ is negligible.

The pedestal density profile is determined by the balance between particle transport and sources, and since we do not have a constraint that gives a quantitative estimate of these two components we have a degree of arbitrariness on the coefficients that define them. To describe the pedestal particle transport we assume that the particle diffusion coefficient is equal to $D_{\mathrm{n} \text {,ped }}=c_{\mathrm{D} / \chi} \chi_{\mathrm{e} \text {,ped }}+D_{\mathrm{n} \text {, neo }}$, where the term $c_{\mathrm{D} / \chi} \chi_{\mathrm{e} \text {,ped }}$ represents the turbulent component of $D_{\mathrm{n} \text {,ped }}$, being proportional to $\chi_{\mathrm{e} \text {,ped }}$ through $c_{\mathrm{D} / \chi}$, and $D_{\mathrm{n} \text {,neo }}$ is the ion neoclassical particle diffusivity. We could have instead used the electron neoclassical particle diffusivity, which is usually lower than that of the ions, and rescaled the coefficients for the source of neutrals and $c_{\mathrm{D} / \chi}$ to obtain the same combination of parametric dependencies and the same pedestal density profiles. However we find that with a larger diffusion coefficient the pedestal density profile evolves faster in time and the convergence of the simulation is quicker. We also assume a fixed pinch velocity $v_{\text {n,ped }}=-0.05 \mathrm{~m} \mathrm{~s}^{-1}$. The values of these coefficients $\left(c_{\mathrm{D} / \chi}=0.03 \mathrm{~m}^{2} \mathrm{~s}^{-1}, v_{\mathrm{n}, \mathrm{ped}}=-0.05 \mathrm{~m} \mathrm{~s}^{-1}\right)$ have been obtained through an optimization procedure trying to match a set of different experimental pedestal density profiles. The resulting effective particle diffusion coefficient is consistent with previous observations from AUG experiments [55]. We underline that the electron heat conductivity $\chi_{\mathrm{e}, \text { ped }}$ is not influenced by these assumptions since it mainly depends on the electron heat source.

A widely applied pedestal model is represented by the EPED model [56], for which it can be interesting to point out the main similarities and differences with respect to our approach. Both EPED and our model scan the pedestal pressure using a transport constraint that relates the pedestal width to its height or gradient, and then use a MHD stability code to find the pedestal structure in the conditions before the type I ELMs onset, given by PBM instability. The difference between the two models is in the different transport constraint, which in EPED is provided by the ballooning critical pedestal technique (BCP), which relates the width of the pedestal $\left(\Delta_{\psi \mathrm{N}}\right)$ to the poloidal beta at the top of the pedestal $\left(\beta_{\mathrm{p}, \text { ped }}\right)$ as $\Delta_{\psi \mathrm{N}}=G \sqrt{\beta_{\mathrm{p}, \text { ped }}}$, where $G$ is a function of collisionality and geometry. This approach allows EPED to be applied as stand-alone, not requiring to be used inside of a transport code. This is possible since it takes the pedestal top electron density as input, and then assumes the same value for the electron and ion temperatures at the pedestal top $T_{\mathrm{e}, \text { ped }}=T_{\mathrm{i} \text {,ped }}$, giving all the elements required to define the pedestal pressure. In contrast, our approach requires the use of a transport code since the adopted constraint does not couple the pedestal width to its height directly, but must solve a system of equations where 
the variables are the heat source, the heat conductivity, the temperature gradient, and the pedestal top temperature. On the other hand, with a transport code we can separately describe transport in all the different channels, allowing us to also predict the density. A comparison on how the two different transport constraints are representative of experimental data is given by figure 12(a) of Ref. [57], where the same pedestals database from figure 2(a) of Ref. [40] is used to show the correlation between the pedestal width $\Delta_{\psi \mathrm{N}}$ and the square root of beta poloidal at the pedestal top $\sqrt{\beta_{\mathrm{p}, \text { ped }}}$. It is interesting to notice that DIII-D and AUG data do not lay on the same region of the graph, perhaps due to the different collisionality regimes between the two different machines, and need a different $G$ coefficient, i.e. steepness of the curve. The transport constraint adopted by the EPED model can be thought as a constraint on the pressure gradient and is associated to a representation of kinetic ballooning modes (KBM). The constraint we impose is instead a critical pedestal averaged electron temperature scale length, which can be interpreted as a $R / L_{\mathrm{T}_{\mathrm{e}}}$ drive for turbulent transport, and therefore can be associated to electron temperature gradient modes (ETG) or micro tearing modes (MTM). This finds some support from Ref. [58], where MTMs are identified as the instability limiting transport in the JET-ILW pedestal, as well as from Ref. [59], where ETGs are found to be plausibly responsible for a significant portion of the electron heat flux in the AUG pedestal. Another observation worth pointing out is that MTMs and ETGs cause a particle diffusion coefficient $D_{\text {n,ped }}$ that is at least one order of magnitude smaller than the electron heat diffusion coefficient $\chi_{\mathrm{e}, \text { ped }}$, as opposed to MHD-like instabilities for which these are comparable [60], and the value of its ratio $D_{\mathrm{n}, \mathrm{ped}} / \chi_{\mathrm{e}, \mathrm{ped}} \sim 0.02-0.06$ is found to be similar to our assumption $\left(c_{\mathrm{D} / \chi}=0.03\right)$.

2.1.3. Core transport To estimate the core turbulent transport fluxes of heat and particles we use the TGLF quasilinear model, with the sat1 [31] version of the saturation rule. The kinetic profiles are simulated using the diagonal terms of the ASTRA transport matrix, that are heat and particle diffusivities $\left(\chi_{\mathrm{e}}, \chi_{\mathrm{i}}, D_{\mathrm{n}}\right)$, plus the particle pinch velocity $\left(C_{\mathrm{n}}\right)$. We use the fluxes calculated by TGLF to compute these coefficients in the core region, and in particular for the particle transport we put the complete TGLF particle flux into the pinch velocity. To account for sawteeth we consider the transport they cause in a "sawtooth time averaged" way. We increase by a fixed amount the heat and particle transport coefficients $\left(\chi_{\mathrm{e}, \mathrm{sr}}=0.2 \mathrm{~m}^{2} / \mathrm{s}\right.$, $\left.\chi_{\mathrm{i}, \mathrm{sr}}=1 \mathrm{~m}^{2} / \mathrm{s}, D_{\mathrm{n}, \mathrm{sr}}=0.5 \mathrm{~m}^{2} / \mathrm{s}\right)$ in the plasma region inside of the inversion radius, so that the resulting kinetic profiles are a time averaged profile over the sawtooth period. We also use a model for the sawtooth crash [61] to take into account the effect of internal kink modes on the current density profile.

In our simulations, the toroidal rotation is not yet evolved in time with an ASTRA transport equation but is taken from the experimental measurements and kept constant. For the majority of cases that we have considered in this work, the rotation is found to have a negligible impact on the other transport channels, as we have verified by comparing results with measured rotation profiles and assumed flat rotation profiles. This result is consistent with usual AUG operation at relatively high density [62]. The addition of a momentum transport equation in the modeling workflow is not expected to be a major challenge, and it is planned to be shortly included for the application to experimental conditions at low density and high NBI heating power. 
2.1.4. Integration of the different components This subsection describes how the transport coefficients in the pedestal and in the core region are combined together. In the ASTRA simulations the extent of the pedestal region is regulated by the value of the pedestal width. In this region the transport coefficients assume the values discussed in section 2.1.2. Conventional core transport modeling of H-mode plasmas applies the boundary condition just inside the pedestal top (usually $\rho_{\text {ped }}^{\text {top }} \sim \rho_{\text {tor }}=0.9$ ). Because the boundary values of temperatures and densities are fixed there, oscillations in the transport coefficients in the peripheral region of the simulation have more limited effects in the predicted behavior of the density and temperature profiles in that region. When the simulation domain is extended up to the last closed flux surface, then any oscillation of the transport coefficients has much stronger effects on the simulated kinetic profiles, rendering the simulations much less stable and robust. For this reason, the radial domain within which the TGLF model has been applied has been limited to $\rho_{\text {tor }}<0.78$. This choice is also motivated by the fact that outside of this region ( $\left.\rho_{\text {tor }}>0.78\right)$ TGLF systematically underpredicts transport, as also observed in Refs. [63, 64], especially in the ion heat channel. For the same reasons we also exclude the region $\rho_{\text {tor }}<0.1$ from the domain of TGLF calculations. The radial domain in which we use TGLF to calculate the turbulent transport fluxes is therefore between $0.1<\rho_{\text {tor }}<0.78$. In the region between the TGLF boundary and the pedestal top location $0.78<\rho_{\text {tor }}<\rho_{\text {ped }}^{\text {top }}$ we have a transition layer in which we include an additional value to the heat and particle transport coefficients $\left(\chi_{\mathrm{e}, \mathrm{tr}}, \chi_{\mathrm{i}, \mathrm{tr}}\right.$, $\left.D_{\mathrm{n}, \mathrm{tr}}\right)$ to ensure smooth gradients, composed of two terms: one that is constant and one that is proportional to the pedestal electron heat diffusion coefficient $\chi_{\mathrm{tr}}=c_{1}+c_{2} \chi_{\text {ped }}$. The values of $c_{1}$ and $c_{2}$ have been chosen in order to obtain gradients of the kinetic profiles, in this region, similar to the experimental ones for the experimental cases considered. Their value is kept constant among the different cases. We find that to obtain temperature gradients similar to the experimental ones in this region the ion heat diffusion coefficient $\chi_{\mathrm{i}, \mathrm{tr}}$ has to be much larger than the one obtained by TGLF at $\rho_{\text {tor }}=0.78$, while for the electrons a heat diffusion coefficient $\chi_{\mathrm{e}, \mathrm{tr}}$ of similar value with respect to the one from TGLF is sufficient. We also find that the ion heat diffusion coefficient $\chi_{\mathrm{i}, \mathrm{tr}}$ has to be generally larger then the one for the electrons $\chi_{\mathrm{i}, \mathrm{tr}}>\chi_{\mathrm{e}, \mathrm{tr}}$. This is in line with the observations reported in Ref. [62], where it is shown that for many AUG cases with different heating schemes, the ion heat flux at the pedestal top was much higher then the electron heat flux. For these reasons the coefficients $c_{1}$ and $c_{2}$ are larger for the ions than for the electrons. For the particle transport we simply assume a fixed diffusion coefficient $D_{\mathrm{n}, \mathrm{tr}}=1.5 \mathrm{~m}^{2} \mathrm{~s}^{-1}$ to ensure low values of the density gradients, as typical of this plasma region. Finally, we add a fixed term to the diffusion coefficients at the separatrix to ensure continuity with the SOL region, where transport is expected to increase due to the open field lines. This also allows us to obtain more realistic gradients of the kinetic profiles with their peak located around the middle of the pedestal, which otherwise would be placed closer to the separatrix.

An important element is the smoothing of the profiles of the transport coefficients, which allows us to obtain smoother and more realistic gradients of the kinetic profiles with respect to the more discontinuous gradients that would result from step-like transport coefficients. This difference is important for the pedestal MHD stability calculation since it uses the profile of the pressure gradients. Figure 4 shows an example of the electron and ion heat diffusion coefficients after (solid) and before (dashed) the smoothing, with an highlight of the components calculated by NCLASS (cyan) and by TGLF (red). The lighter dotted red line represents TGLF solution in the edge region, which corresponds to very large electron heat fluxes and practically zero 

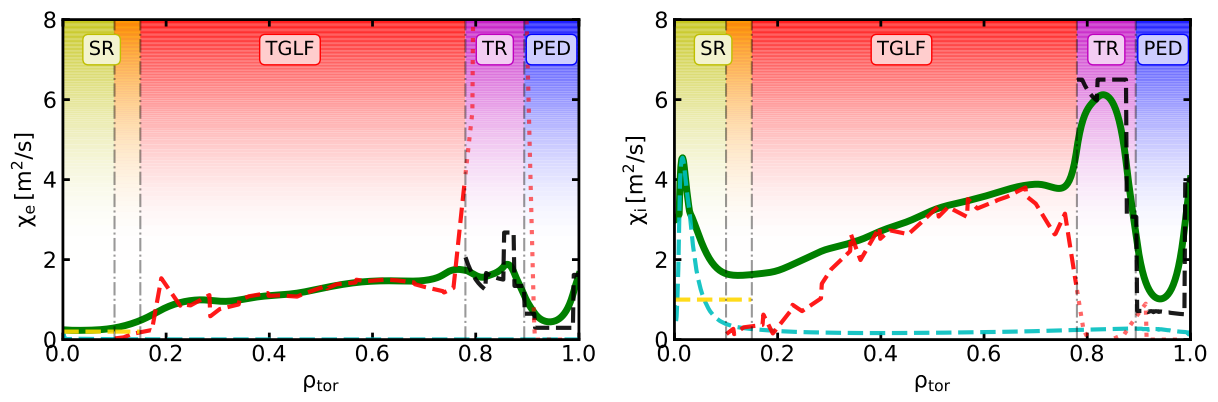

Figure 4. Example of electron and ion heat diffusion coefficients, separated in the components corresponding to the different plasma regions. The figure also illustrates the profiles before (dashed) and after (solid) the smoothing, with an highlight of the components calculated by NCLASS (cyan) and by TGLF (red), and of the component added to account for the transport caused by sawteeth. The lighter dotted red line shows what solution would TGLF give in the edge region.

ion heat fluxes. The yellow dashed line represents the additional transport diffusivity that we use to describe the sawteeth. It is also shown the separation between the sawtooth region (yellow), the TGLF computational domain (red), the aforementioned transition region (magenta), and the pedestal region (blue). The orange area highlights the fact that the sawtooth and the TGLF regions overlap.

The ASTRA simulations are performed using a linear radial grid consisting of 601 points. Such a large number of points is necessary to be able to resolve the pedestal with a definition that is large enough to perform a fine scan of the pedestal width, otherwise the final result of the workflow would have a too large uncertainty in the pedestal top pressure and therefore in the stored energy and energy confinement time. The time-step in ASTRA is 2ms, and TGLF is called every $10 \mathrm{~ms}$, which is a value that finds a good compromise between computational cost and stability of the kinetic profiles due to the aforementioned TGLF oscillations. Every ASTRA simulation requires $\sim 4 \mathrm{~h}$ of computing time running in parallel on 16 cores on a single CPU. The simulations run for $4 \mathrm{~s}$ in the ASTRA time frame (2000 iterations and 400 TGLF calculations for each of the 64 radial location at which it is called), which we find is a sufficient time to obtain stationary profiles with excellent match between the fluxes and the volume integral of the sources.

\subsection{Pedestal MHD stability}

The pressure and current density profiles corresponding to the different values of the pedestal width from the scan are passed to the HELENA high resolution equilibrium solver [65], for a finer calculation of the equilibrium with respect to the faster but lower resolution SPIDER calculation used in ASTRA. The stability of each of these equilibria is tested by using the MISHKA linear MHD stability code for a range of modes $(1<n<70)$. We use the MISHKA-1 variant of the code, since we noticed that for our application it is more robust against numerical inaccuracies with respect to the MISHKA-fast solver [37]. The MISHKA-fast version reduces the computational cost by allowing for a radially varying range of poloidal mode numbers, which can be particularly efficient when analyzing higher toroidal mode numbers, but in fact we 
did not noticed an appreciable difference in computing time, that is usually $\sim 1$ hour running on a single CPU core. We set a critical growth rate of $\gamma=0.04 \gamma_{\text {Alfven }}$ for determining the pedestal stability limit. We select as result of the modeling workflow the point from the scan with the highest pedestal pressure and width that is still PBM stable $\left(\gamma<0.04 \gamma_{\text {Alfven }}\right)$.

\subsection{Assumptions}

\begin{tabular}{|c|c|c|}
\hline assumption & determination & general/machine specific \\
\hline$T_{\text {e,sep }}$ (eq. 5 from $[43]$ ) & theoretical & general \\
\hline$n_{\mathrm{e}, \mathrm{sep}}($ eq. 8 from [43]) & theoretical & attached divertor conditions \\
\hline$T_{\mathrm{i}, \mathrm{sep}}=2 T_{\mathrm{e}, \mathrm{sep}}$ & heuristic & to be studied \\
\hline$\gamma=6, \alpha=3.3^{\circ}, Z_{\mathrm{eff}, \mathrm{div}}=1.3$ & typical parameters & AUG DivIII specific \\
\hline$p_{0}($ eq. 2$)$ & empirical & AUG DivIII specific \\
\hline$f_{\mathrm{R}}=0.8$ & heuristic & metallic wall specific \\
\hline$c_{\mathrm{div}, \mathrm{wall}}=0.0168$ & heuristic & AUG DivIII specific \\
\hline$A_{\mathrm{eff}}=A_{\mathrm{LCFS}} / 3$ & heuristic & to be studied \\
\hline$\alpha_{\text {wall,sep }}($ eq. 6$)$ & theoretical & deuterium specific \\
\hline$T_{0}=5 \mathrm{eV}$ & heuristic & general \\
\hline$<\nabla T_{\mathrm{e}}>/ T_{\mathrm{e}, \mathrm{ped}}=0.5$ & empirical & general \\
\hline$\Delta_{\mathrm{T}_{\mathrm{e}}}=\Delta_{\mathrm{T}_{\mathrm{i}}}=\Delta_{\mathrm{n}_{\mathrm{e}}}$ & heuristic & general \\
\hline$\chi_{\mathrm{i}, \text { ped }}=\chi_{\mathrm{e}, \mathrm{ped}}+\chi_{\mathrm{i}, \text { neo }}$ & heuristic & general/to be tested \\
\hline$D_{\mathrm{n}, \mathrm{ped}}=c_{\mathrm{D} / \chi} \chi_{\mathrm{e}, \mathrm{ped}}+D_{\mathrm{n}, \mathrm{neo}}$ & heuristic & general/to be tested \\
\hline$c_{\mathrm{D} / \chi}=0.03$ & heuristic & general/to be tested \\
\hline$v_{\mathrm{n}, \mathrm{ped}}=-0.05 \mathrm{~m} \mathrm{~s}^{-1}$ & heuristic & general/to be tested \\
\hline$\chi_{\mathrm{e}, \mathrm{sr}}=0.2 \mathrm{~m}^{2} / \mathrm{s}$ & heuristic & general/to be tested \\
\hline$\chi_{\mathrm{i}, \mathrm{sr}}=1 \mathrm{~m}^{2} / \mathrm{s}$ & heuristic & general/to be tested \\
\hline$D_{\mathrm{n}, \mathrm{sr}}=0.5 \mathrm{~m}^{2} / \mathrm{s}$ & heuristic & general/to be tested \\
\hline$c_{\mathrm{W}}=2 \times 10^{-5}$ & heuristic & to be studied \\
\hline
\end{tabular}

Table 1. The first column summarizes the assumptions taken into the model. The second column contains the information on how the assumptions were determined. The third column describes the validity of the assumptions.

Table 1 summarizes all the assumptions introduced into the model. The numerical values listed in the first column are kept constant for all of the simulations performed on the experimental cases considered in this work. The second column describes how the different assumptions were determined, that is if they are theory based, empirical (derived from regressions on experimental data), or heuristic (assumptions for which we do not have enough constraints, so they were determined trying to reproduce the experiments, while still being compatible with the theory of the underlying physics). The empirical and heuristic elements contained in the model have to be tested and generalized to extend the applicability to different experimental conditions. The third column contains the information on the validity of the assumptions, that is if they are expected to be valid also for other machines or if they are AUG specific (in the configuration with the DivIII divertor), if they are valid only for some particular type of operation (e.g. with deuterium, for attached divertor conditions), or if their validity needs to be investigated. The parameters for which we expect the strongest machine dependency are the parameters related to the divertor and SOL, since the materials and the geometry of the wall and the divertor can change significantly between different 
machines. These are in particular the scaling of the divertor neutral pressure $p_{0}$, and the coefficients related to the source of neutrals $\left(f_{\mathrm{R}}, c_{\mathrm{div} \text {,wall }}, A_{\mathrm{eff}}\right)$. For the $p_{0}$ scaling a new derivation of the scaling for the tokamak of interest would be needed, and in lack of experimental information, synthetic data from simulations could be used. This approach could also be applied to future devices. Also the recycling fraction coefficient $f_{\mathrm{R}}$ depends on the material of the wall of the machine, and is expected to be smaller for carbon walled devices. All the other parameters are either derived from theoretical expressions, or are known parameters of the machine.

We performed a sensitivity study on one experimental case to test how a certain variation of the parameters listed in table 1 affects the final result of the model. A critical assumption in the model is $\left\langle\nabla T_{\mathrm{e}}\right\rangle / T_{\mathrm{e} \text {,ped }}=0.5$, which is derived from experimental data, and can therefore contain uncertainties. The deviation in the database used to obtain $\left\langle\nabla T_{\mathrm{e}}\right\rangle / T_{\mathrm{e} \text {,ped }}=0.5$ is around $10 \%$. With a change of $10 \%\left(<\nabla T_{\mathrm{e}}>/ T_{\mathrm{e}, \text { ped }}=0.5 \pm 0.05\right)$ the change in the plasma thermal energy was $\Delta W_{\text {th }}=3.5 \%$, so we conclude that such uncertainties do not have an important impact on the final result of the model. With a change of $10 \%$ among the other different parameters the observed change in the plasma thermal energy was always lower than $\Delta W_{\text {th }}<3.5 \%$. This small sensitivity on the assumptions is due to the fact that the pedestal stability calculation is very robust. Small changes caused by variations in the parameters of the model do not cause a significant change in the predicted pedestal top pressure, and consequent small difference in the profiles in the pedestal layer do not significantly affect the core profiles and therefore the stored energy. External actuators, or the inputs of the model (e.g. the heating power) produce much larger changes in the pedestal structure (top pressure and gradients), and result therefore in a substantially different pedestal and global confinement.

Another important aspect is the relationship between the pedestal width and the pedestal top pressure that results from imposing $\left\langle\nabla T_{\mathrm{e}}\right\rangle / T_{\mathrm{e} \text {,ped }}=0.5$. One might reach the wrong conclusion that $\left\langle\nabla T_{\mathrm{e}}\right\rangle=T_{\mathrm{e} \text {,ped }} / \Delta_{\text {ped }}$, and therefore $T_{\mathrm{e}, \text { ped }} / \Delta_{\text {ped }} / T_{\mathrm{e}, \mathrm{ped}}=1 / \Delta_{\text {ped }}=$ constant. However, this is wrong, because $\left\langle\nabla T_{\mathrm{e}}\right\rangle=$ $\left(T_{\mathrm{e}, \mathrm{ped}}-T_{\mathrm{e}, \mathrm{sep}}\right) / \Delta_{\text {ped }}$, which does not lead to a constant value of the pedestal width.

\section{Experimental results considered for the first application of the model}

This modeling framework is tested by simulating AUG discharges. We selected 10 different time windows of stationary conditions from a total of 4 different discharges in order to have a reasonably wide range of parameter variations. Table 2 gives an overview of the main plasma parameters for the discharges taken into consideration. In this database the magnetic field is constant $B=-2.5 \mathrm{~T}$, and we have selected a variation in plasma current $I_{\mathrm{p}}=0.6-1 \mathrm{MA}$, heating power $P=4.5-12 \mathrm{MW}$, and fueling rate $\Gamma_{\mathrm{D}}=0.15-2 \times 10^{22} \mathrm{e} / \mathrm{s}$. All these experimental cases have similar plasma shape, with a relatively low plasma triangularity, typical of AUG. In these discharges on-axis ECRH was used to avoid tungsten accumulation. The values for the effective charge $Z_{\text {eff }}$ are calculated using the Integrated Data Analysis (IDA) [66]. This selection allows us to test the model on the variation of parameters which are included in the IPB98,(y2) scaling law (heating power, and plasma current), with in addition the possibility of investigating whether the model can capture the effect of fueling on confinement. 
Integrated modeling of ASDEX Upgrade plasmas combining core, pedestal and scrape-off layer physics16

\begin{tabular}{cccccccccccc}
\hline shot & $\begin{array}{c}\text { time } \\
{[\mathrm{s}]}\end{array}$ & $\begin{array}{c}B_{\mathrm{t}} \\
{[\mathrm{T}]}\end{array}$ & $\begin{array}{c}I_{\mathrm{p}} \\
{[\mathrm{A}]}\end{array}$ & $\begin{array}{c}P_{\mathrm{NBI}} \\
{[\mathrm{MW}]}\end{array}$ & $\begin{array}{c}P_{\mathrm{ECRH}} \\
{[\mathrm{MW}]}\end{array}$ & $q_{95}$ & $\begin{array}{c}\bar{n}_{\mathrm{e}} \\
{\left[10^{19} / \mathrm{m}^{3}\right]}\end{array}$ & $\begin{array}{c}\Gamma_{\mathrm{D}} \\
{\left[10^{22} \mathrm{e} / \mathrm{s}\right]}\end{array}$ & $\begin{array}{c}\Gamma_{\mathrm{N} 2} \\
{\left[10^{22} \mathrm{e} / \mathrm{s}\right]}\end{array}$ & $Z_{\text {eff }}$ & color \\
\hline 34153 & 2.0 & 2.5 & 0.6 & 10 & 1.77 & 7.1 & 4.8 & 0.46 & 0 & 1.80 & $\mathbf{\square}$ \\
33173 & 2.7 & 2.5 & 1 & 10 & 2.00 & 4.0 & 6.3 & 0.46 & 0 & 1.45 & $\square$ \\
33173 & 3.7 & 2.5 & 1 & 10 & 2.00 & 4.0 & 7.0 & 1.00 & 0 & 1.35 & $\square$ \\
33173 & 4.7 & 2.5 & 1 & 10 & 2.00 & 4.0 & 7.6 & 1.88 & 0 & 1.30 & $\square$ \\
32201 & 2.9 & 2.5 & 1 & 5 & 1.35 & 4.0 & 7.3 & 0.96 & 0 & 1.15 & $\square$ \\
32201 & 3.7 & 2.5 & 1 & 5 & 1.35 & 4.0 & 7.7 & 1.90 & 0 & 1.15 & $\square$ \\
32201 & 4.9 & 2.5 & 1 & 7.5 & 1.35 & 4.0 & 7.7 & 1.90 & 0 & 1.15 & $\square$ \\
32201 & 5.7 & 2.5 & 1 & 7.5 & 1.35 & 4.0 & 7.3 & 0.96 & 0 & 1.15 & $\square$ \\
33616 & 5.2 & 2.5 & 0.8 & 5 & 1.16 & 5.2 & 6.1 & 0.35 & 0 & 1.12 & $\square$ \\
33616 & 7.2 & 2.5 & 0.8 & 2.5 & 1.63 & 5.2 & 6.1 & 0.15 & 0 & 1.12 & $\square$ \\
\hline
\end{tabular}

Table 2. Summary of the experimental cases considered. The columns contain the shot number, the central time of the considered time-window, the toroidal magnetic field, the plasma current, the NBI power, the edge safety factor, the line averaged density, the deuterium fueling rate, the effective charge, and the color used as a reference for the other figures. The first two lines correspond to the two cases representing a scan in plasma current. Lines 2 to 4 represent a fueling scan. Lines 3,5 , and 8 represent a power scan at constant fueling, as also lines 4,6 , and 7 but at a higher fueling level. Lines 9 and 10 represent another power scan, at lower plasma current and with fixed line averaged density instead of fixed fueling rate.

\section{Results from model application}

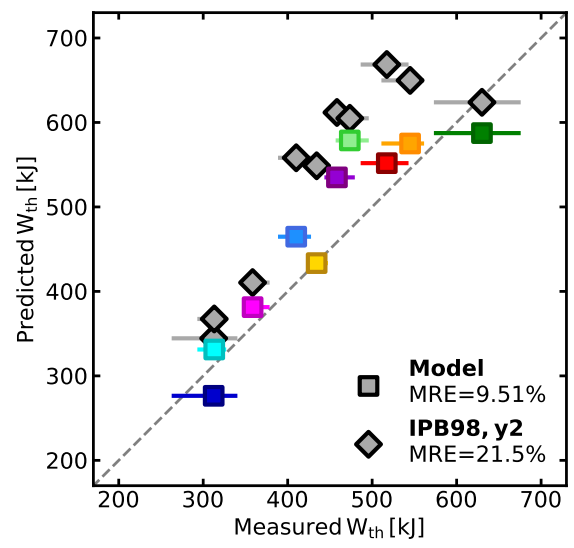

Figure 5. Comparison of the measured thermal energy with the prediction of the model (colored squares), and IPB98(y,2) (gray diamonds). The different colors of the squares refer to the different entries in table 2.

Figure 5 shows a comparison of the measured thermal energy with the prediction of the model (colored squares), and IPB98 (y,2) (gray diamonds). The experimental thermal stored energies have been calculated as the average between the values obtained using different techniques. These are: the integral of the experimental kinetic profiles obtained in the stationary phase before an ELM crash, and $W_{\mathrm{MHD}}-W_{\text {fast }}$, where $W_{\mathrm{MHD}}$ is the plasma energy obtained from the equilibrium reconstruction and $W_{\text {fast }}$ 
is the energy of the fast ions. The error-bars represent the maximum and minimum values obtained using the different techniques, and include the uncertainty on the calculation of the fast ions energy, and the one given by the ELMs filtering. In the experimental cases considered in this work the time averaged plasma energy content calculated over multiple ELMs cycles differs from that just before the ELMs crash by less than $2 \%$. This difference is negligible with respect to the error-bars of the measurements and with respect to the differences among the various predictions. It is therefore acceptable to compare the time averaged thermal energy predicted by the IPB98 $(y, 2)$ with the thermal energy just before the ELMs onset predicted by the model and calculated from experimental data. The electron temperature and density profiles are given by the Integrated Data Analysis (IDA) [67]. For the ion temperature profiles we use the data provided by the charge exchange recombination spectroscopy (CXRS) diagnostics, fitted with the gaussian process regression (GPR) techniques presented in Ref. [68].

The mean relative error for the model $M R E=9.51 \%$ suggests that the prediction is accurate in reproducing the change in energy confinement caused by the different parameters of plasma operation, and is more accurate with respect to the IPB98 $(y, 2)$ scaling law $M R E=21.5 \%$. We underline that no boundary condition is taken from the measurements of the kinetic profiles in the ASTRA simulations, thereby to large extent we can consider that the two approaches use a similar set of input parameters, with the exception of $Z_{\text {eff }}$, which is required by our model. Some of the engineering parameters (heating power and plasma current) are closely and directly correlated to plasma confinement, and therefore a statistical regression (i.e. a scaling law) can robustly describe these dependencies. However, this kind of approach is limited by the fact that some other engineering parameters (e.g. the fueling rate) do not exhibit a direct correlation with confinement, and it is therefore very difficult to include the effect of such hidden parameters into a multivariate regression. This implies that the predictions of scaling laws become less reliable when there is a strong variation in this kind of parameters, as in this case for the IPB98 $(\mathrm{y}, 2)$ where the main reason for the large MRE is the relatively large values of the fueling rate. Our model instead captures the dependence of confinement on the engineering parameters by giving a description of the physics that regulates these effects, by combining theoretical models and empirical elements. This allows us to capture the dependencies on operational parameters beyond the capabilities of scaling laws, as long as the description of their behaviors is included in the model. As a result the model is more accurate then the IPB98 $(y, 2)$ for these cases, and more in general we can expect that it will also be more precise for a larger number of cases if the theoretical models and the empirical elements adopted are reliable and general enough to cover the new regions of the parameters space in which they are applied.

Figure 6 shows a comparison between the predicted and measured values of $T_{\mathrm{i}} / T_{\mathrm{e}}$ at the pedestal top (left) and at the center of the plasma, at $\rho_{\text {tor }}=0.1$ (right). We chose this radial location instead of $\rho_{\text {tor }}=0$ since at the magnetic axis some of the experimental measurements are missing and fitted profiles result from extrapolations. The model can well reproduce the values of $T_{\mathrm{i}} / T_{\mathrm{e}}$ at the pedestal top. This is an important result, and is an improvement over the EPED model, which assumes $T_{\mathrm{e}, \mathrm{ped}}=T_{\mathrm{i}, \mathrm{ped}}$. As described in 4.4, $T_{\mathrm{e} \text {,ped }}$ is underestimated for the case at $I_{\mathrm{p}}=0.6[M A]$ (blue), and therefore it exhibits a larger error respect to the other cases. Also the values of $T_{\mathrm{i}} / T_{\mathrm{e}}$ at the center of the plasma are well reproduced by the model.

Figure 6 shows a comparison between the predicted and measured values of $T_{\mathrm{i}}$ (empty squares) and $T_{\mathrm{e}}$ (full diamonds) at the center of the plasma, at $\rho_{\mathrm{tor}}=0.1$ 

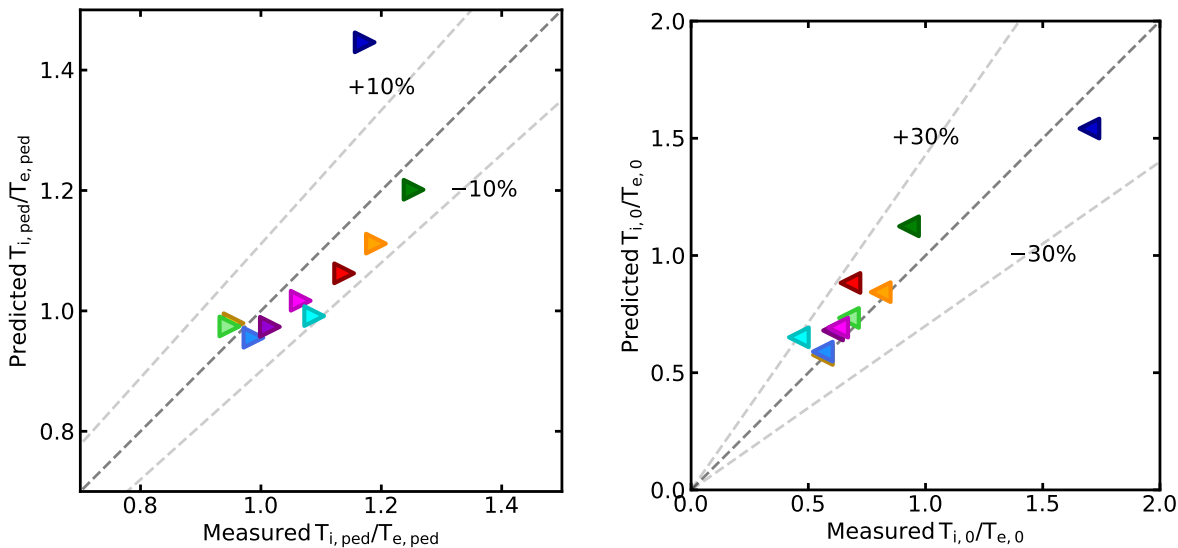

Figure 6. Comparison between the predicted and measured values of $T_{\mathrm{i}} / T_{\mathrm{e}}$ at the pedestal top (left) and at the center of the plasma, at $\rho_{\text {tor }}=0.1$ (right). The different colors of the symbols refer to the different entries in table 2 .
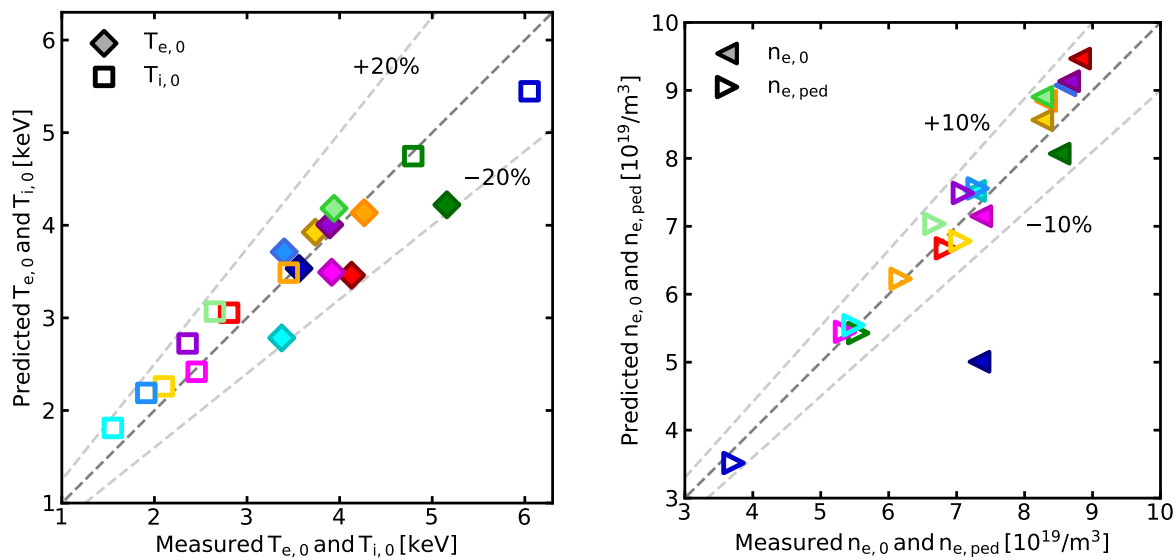

Figure 7. Comparison between the predicted and measured values of $T_{\mathrm{i}}$ (empty squares) and $T_{\mathrm{e}}$ (full diamonds) at the center of the plasma, at $\rho_{\text {tor }}=0.1$ (left), and (rigth) of $n_{\mathrm{e}}$ at the pedestal top (empty triangles) and $n_{\mathrm{e}}$ at the center of the plasma (full triangles). The different colors of the symbols refer to the different entries in table 2.

(left), and (rigth) of $n_{\mathrm{e}}$ at the pedestal top (empty triangles) and $n_{\mathrm{e}}$ at the center of the plasma (full triangles). An important aspect is that the model can correctly predict the pedestal top electron density. This is another improvement over the EPED model, which lacks the capability of predicting the pedestal top electron density, as this must be given as input. 

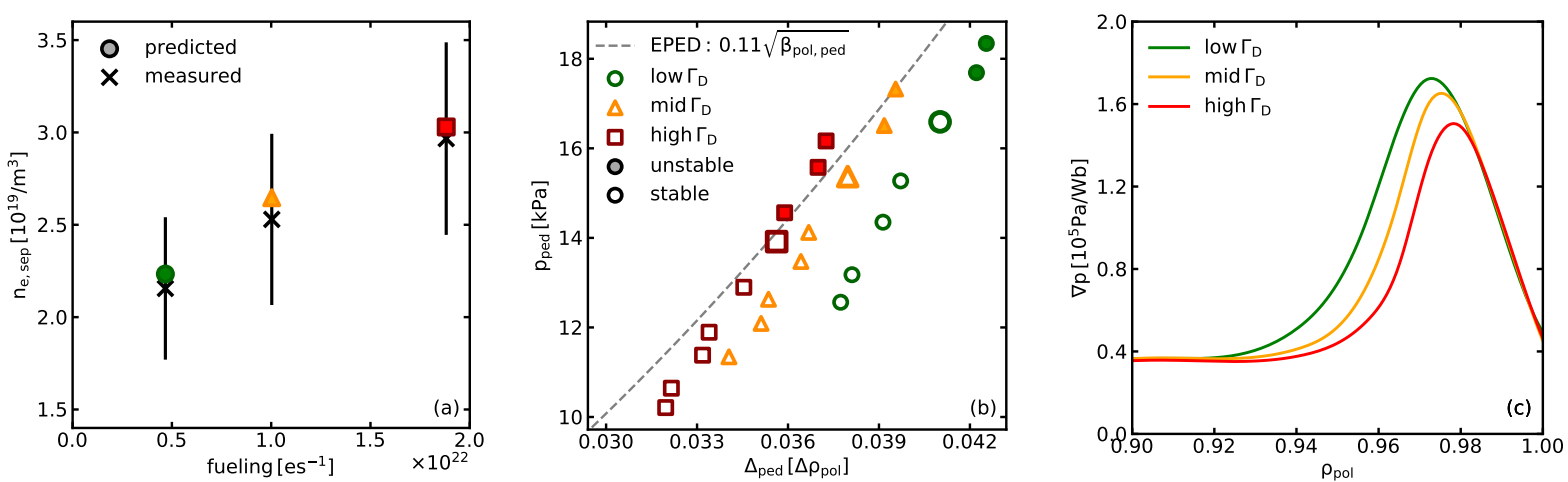

Figure 8. a) Predicted (filled symbols) and measured (crosses) separatrix electron density as a function of the fueling rate. b) ASTRA pedestal width scan for the 3 fueling rate levels. The empty symbols correspond to PB stable pedestal conditions as predicted by the MISHKA MHD stability calculation, while the filled symbols correspond to unstable conditions (i.e. ELM onset). The dashed line represents the solution that would be provided by EPED's BCP technique, assuming a fixed value, typical of AUG, of the proportionality term between $\Delta_{\psi \mathrm{N}}$ and $\sqrt{\beta_{\mathrm{p}, \mathrm{ped}}}$. c) Pressure gradients profiles corresponding to the highest stable pedestal pressure.

\subsection{Fuelling scan}

Among these cases, we focus on the stationary phases of the AUG discharge \#33173, a gas puff scan at $B=-2.5 \mathrm{~T}, I_{\mathrm{p}}=1 \mathrm{MA}, P=13 \mathrm{MW}$, that is particularly interesting because it features the typical confinement degradation with increasing gas puff, observed in AUG, JET-ILW and TCV [69-71]. This effect is clearly not captured by the IPB98 scaling law. The reduction in pedestal and global confinement has been related to an outward shift of the density profile, which appears together with an increased value of the separatrix density [69]. Figure 8 (a) shows that the predicted separatrix density is in excellent agreement with the measurements, evaluated with the IDA, and the effect of fueling is well captured. In AUG experiments it has been observed that the separatrix density is strongly related to the divertor neutral pressure [49], which increases with increasing fueling rate. The SOL model we included in ASTRA allows us to describe how an increase in the fueling rate causes an increase in the divertor neutral pressure (eq. 2) and ultimately how an increase in the divertor neutral pressure causes an increase in the separatrix density (eq. 8 from [43]). Figure 8 (b) shows the scans in pedestal width, where the filled symbols correspond to PBM unstable conditions, while the open ones represent stable conditions. The larger open symbols identify the highest stable pedestal pressure, which correspond to the final result of the model for the pedestal prediction. As one can see the predicted pedestal pressure decreases with increasing fueling rate $\left(\Gamma_{\mathrm{D}}\right)$. One can also see that the same pedestal pressure among the 3 different cases (low, medium, and high fueling) corresponds to different values of pedestal widths, and therefore the same pedestal width among the 3 different cases corresponds to different values of pedestal pressure. This is because, in the ASTRA prediction of the kinetic profiles, when the fueling rate is increased, the separatrix density and then the whole pedestal density profile increase. 
For the same value of the pedestal width, the electron temperature at the pedestal top is similar among the 3 different cases due to the constraint $\left\langle\nabla T_{\mathrm{e}}\right\rangle / T_{\mathrm{e}, \mathrm{ped}}=0.5$, while the pedestal top density is higher with higher fueling. Therefore, for the same value of the pedestal width, the pedestal top pressure is higher with higher fueling, or, for the same value of the pedestal pressure, the pedestal width is smaller with higher fueling. Since the peak of the pressure gradient is located approximately in the middle of the pedestal width, this moves outwards with higher fueling. Because the ballooning stability is sensitive to the location of this peak (the closer to the separatrix the more it is unstable), the higher fueling case will be limited to a lower value of the pressure gradient, because of the lower ballooning stability limit. This is illustrated in figure 8 (c), which shows the pressure gradient profiles of the highest stable pedestals for the 3 different cases.

As depicted by the gray dashed line in figure 8 (b), with the transport assumption of the EPED [56] model, assuming a fixed value (0.11, typical of AUG) of the proportionality term between $\Delta_{\psi \mathrm{N}}$ and $\sqrt{\beta_{\mathrm{p}, \text { ped }}}$, the three cases would have the same pedestal pressure at the same pedestal width, being all aligned on the same line. This would mean that the peak of the pressure gradients would be located at a similar position, obtaining a similar ballooning stability limit. The change of the value of the pedestal top density alone would not cause a large change in the predicted pedestal pressure. The EPED model would therefore predict a similar value of the pedestal pressure for the 3 different fueling levels. One should note that this figure does not provide any information on what would be the pedestal pressure predicted by EPED for these cases, it only illustrates the dependence of the pedestal pressure on the width for the two different transport constraints $\left(\Delta_{\psi \mathrm{N}}=0.11 \sqrt{\beta_{\mathrm{p}, \mathrm{ped}}}\right.$ and the one resulting from $\left.<\nabla T_{\mathrm{e}}>/ T_{\mathrm{e}, \mathrm{ped}}=0.5\right)$.
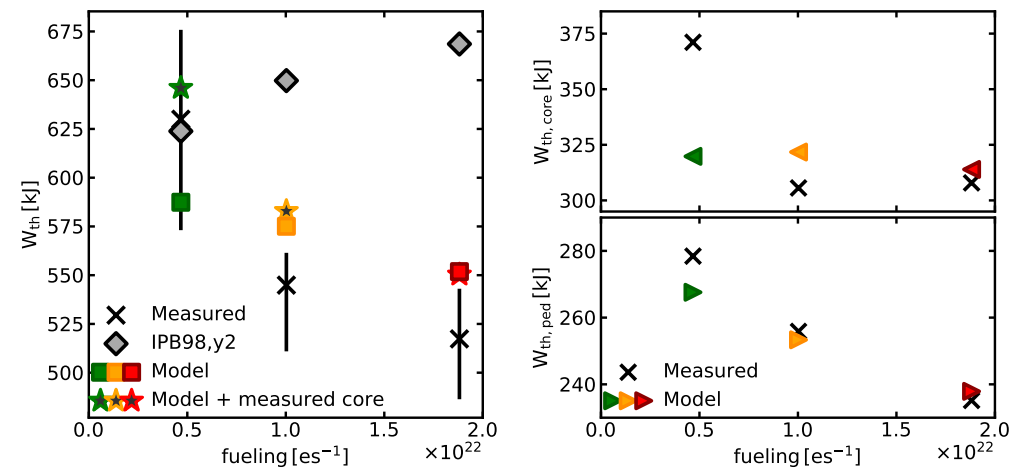

Figure 9. Thermal energy predicted by the model (colored squares), by the IPB98 (y,2) (gray diamonds), and measured (black crosses) as a function of fueling rate (left). The colored stars with black filling show the thermal energies obtained by combining the predicted pedestal profiles with the experimental core profiles. Experimental and predicted thermal energy separated in the pedestal and core components (right).

Figure 9 shows the thermal energy predicted by the model (colored squares), compared to the measurements (black crosses) for the three different cases. We also show the experimental and predicted thermal energy separated in the pedestal and core components, from which we can see that for the lowest fueling case (in green) 
Integrated modeling of ASDEX Upgrade plasmas combining core, pedestal and scrape-off layer physics 21
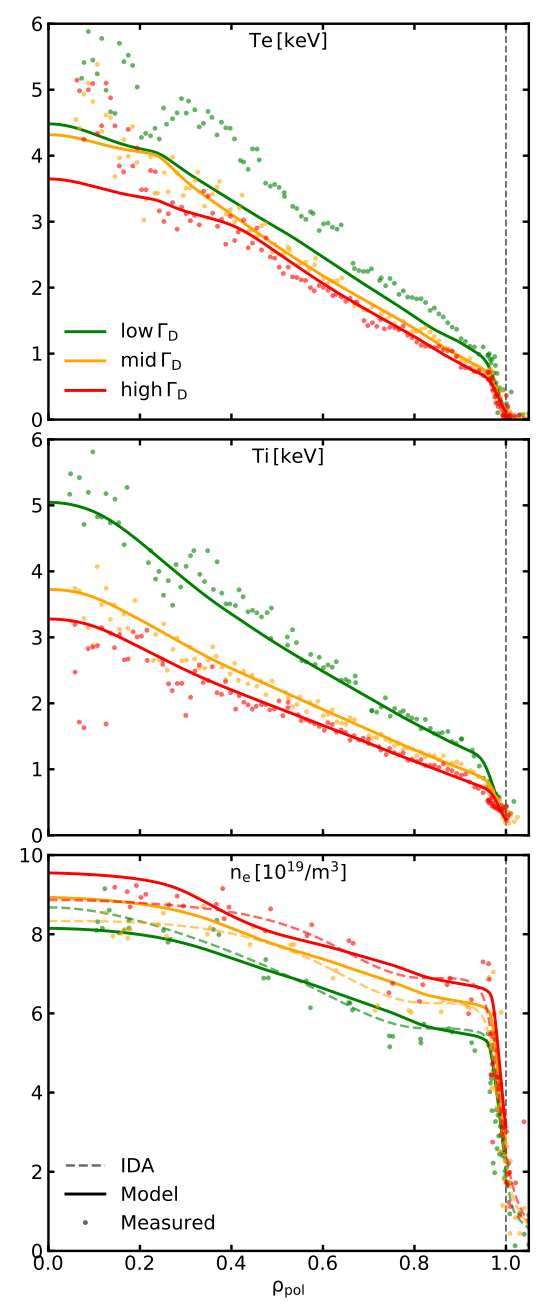

Figure 10. Experimental measurements (dots), and predicted profiles (lines) of electron and ion temperature, and electron density for the different fueling rates. The dashed lines show the electron density profile obtained with the Integrated Data Analysis (IDA).

there is a large disagreement between the predicted and the measured core thermal energy. This is because for this case TGLF overestimates the electron heat transport, as depicted in Figure 10 where it can be seen that the predicted electron temperature (solid line) is lower then the experimental measurements (dots). The colored stars with black filling show the thermal energies obtained by combining the predicted pedestal profiles with the experimental core profiles, which are in good agreement with the measurements. This highlights the accurate prediction of the pedestal. The IPB98 $(y, 2)$ scaling law (gray diamonds) fails to capture this effect, and in contrast to the experimental trend, predicts an increasing stored energy with increasing fueling, as a consequence of the positive dependence of the confinement time on the line averaged density in $\operatorname{IPB} 98(\mathrm{y}, 2)$. 
Integrated modeling of ASDEX Upgrade plasmas combining core, pedestal and scrape-off layer physics 22

\subsection{Power scan at high fueling}

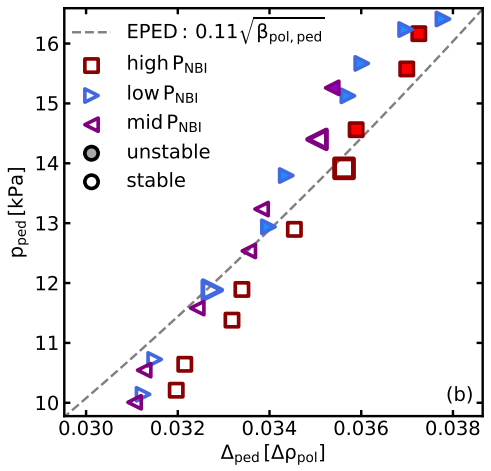

Figure 11. ASTRA pedestal width scan for the 3 time windows corresponding to a heating power scan. The empty symbols correspond to PB stable pedestal conditions as predicted by the MISHKA MHD stability calculation, while the filled symbols correspond to unstable conditions (i.e. ELM onset). The dashed line represents the solution that would be provided by EPED's BCP technique, assuming a fixed value, typical of AUG, of the proportionality term between $\Delta_{\psi \mathrm{N}}$ and $\sqrt{\beta_{\mathrm{p}, \mathrm{ped}}}$.

Combining different stationary phases from discharges \#33173 and \#32201 we obtain two power scans at two different fueling levels. Figure 11 shows the pedestal width scans for the three different levels of NBI power at the highest fueling rate (\#33173 at $4.7 \mathrm{~s}, \# 32201$ at $3.7 \mathrm{~s}$ and at $4.9 \mathrm{~s}$ ). As one can see the curves corresponding to the three different power levels do not exhibit a large offset like in the fueling scan case, since the fueling rate is constant and therefore there is no evident shift of the density profile.
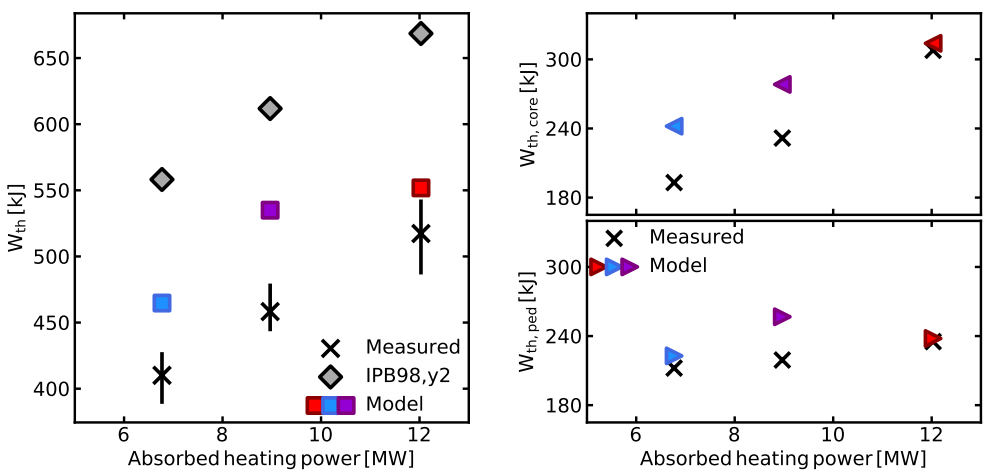

Figure 12. Thermal energy predicted by the model (squares), by the IPB98(y,2) (diamonds), and measured (black crosses) as a function of the heating power (left). Experimental and predicted thermal energy separated in the pedestal and core components (right). 
Integrated modeling of ASDEX Upgrade plasmas combining core, pedestal and scrape-off layer physics 23

Figure 12 shows that the predicted pedestal pressure is in good agreement with the measurements, except for the case corresponding to the medium power level, for which the pedestal pressure is overpredicted. The core pressure is overpredicted by TGLF for the two cases with the lowest power levels. Overall, the dependence of heating power on the total thermal energy is well captured, and the prediction of the model is more accurate with respect to the one of the IPB98 $(y, 2)$ scaling law. Interestingly the different levels of heating power have a much stronger effect on the core stored energy than on the pedestal. The results are similar also for the other power scan at lower fueling rate.

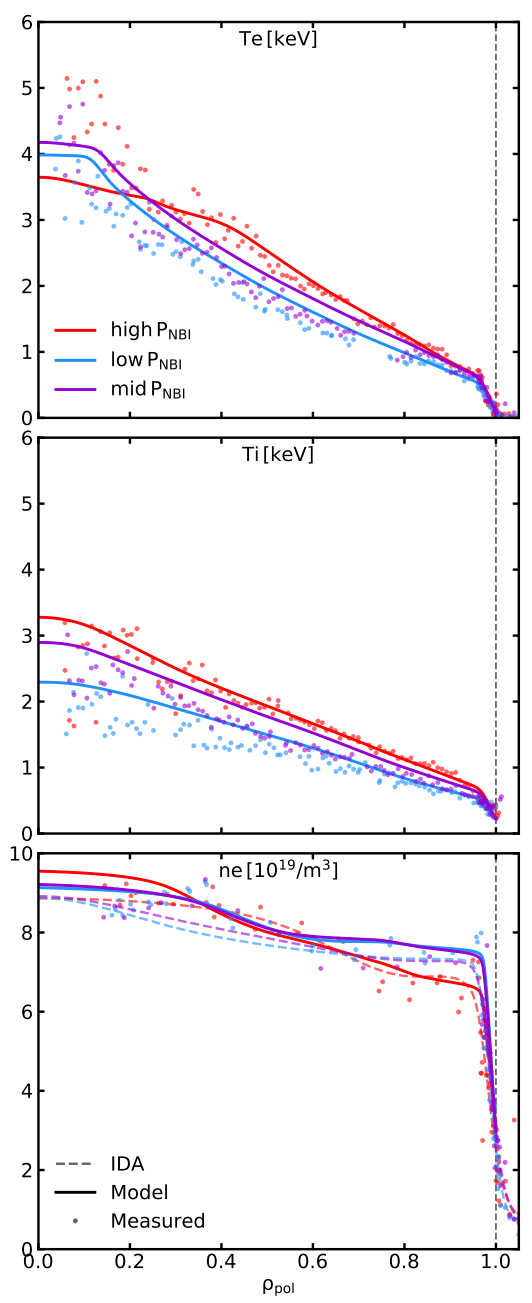

Figure 13. Experimental measurements (dots), and predicted profiles (lines) of electron and ion temperature, and electron density for the different levels of heating power. The dashed lines show the electron density profile obtained with the Integrated Data Analysis (IDA).

The kinetic profiles are shown in figure 13, where it can be seen that the predicted density profiles match the experimental ones, reproducing well the peaking. The 
electron and ion temperatures are slightly overpredicted for the lower heating power cases, with their gradients starting to deviate from the experimental ones at around $\rho_{\text {pol }} \sim 0.8$, the transport short-fall region of TGLF.

\subsection{Power scan at fixed line averaged density}

Another heating power scan is provided by the two stationary phases of the discharge \#33616. In this case we scan the heating power while keeping all the other parameters of the IPB98 $(\mathrm{y}, 2)$ scaling law fixed, in facts in this discharge the line averaged density is constant, achieved using gas puff as feedback. Another difference with respect to the previously discussed cases is in the plasma current $I_{\mathrm{p}}=0.8 \mathrm{MA}$. The two different time windows analyzed have different heating power levels, the first with $\sim 6.5 \mathrm{MW}$ $(5 \mathrm{MW} \mathrm{NBI}+1.16 \mathrm{MW}$ ECRH$)$, and the second with $\sim 4.5 \mathrm{MW}(2.5 \mathrm{MW} \mathrm{NBI}+$ 1.63MW ECRH). This discharge has a relatively low ELM frequency and very good quality measurements, which allows us to perform an accurate comparison between the predicted and the measured kinetic profiles in the pedestal.
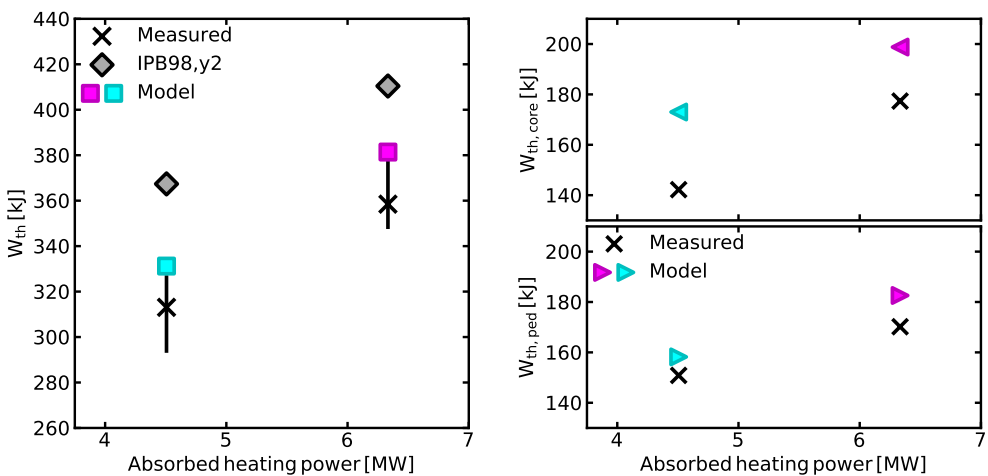

Figure 14. Thermal energy predicted by the model (squares), by the IPB98 $(y, 2)$ (diamonds), and measured (black crosses) as a function of the heating power (also the fueling rate is slightly changed, while the line averaged density is constant) (left). Experimental and predicted thermal energy separated in the pedestal and core components (right).

The thermal energy predicted for these cases is shown in figure 14. The prediction of the model is in very good agreement with the measurements, and surprisingly is more accurate with respect to the $\operatorname{IPB} 98(\mathrm{y}, 2)$, since the scaling law is usually very robust for such low levels of fueling (in this case required to obtain the requested line averaged density). The mismatch between the predictions and the measurements is mostly associated to the core, again due to underestimated heat transport by TGLF in the region around $\rho_{\text {pol }} \sim 0.8$, as it can be noticed by looking at the temperature profiles in figure 15, while the pedestal is accurately predicted. The change in stored energy with different heating powers is well captured.

An interesting aspect highlighted by this case is the role of particle transport and sources in determining the pedestal density profile. Since the core density peaking is practically identical in the two different time windows, the constant line averaged density results in a constant density at the pedestal top. The required fueling rate in the time window with the lowest heating power level is extremely low 
Integrated modeling of ASDEX Upgrade plasmas combining core, pedestal and scrape-off layer physics25
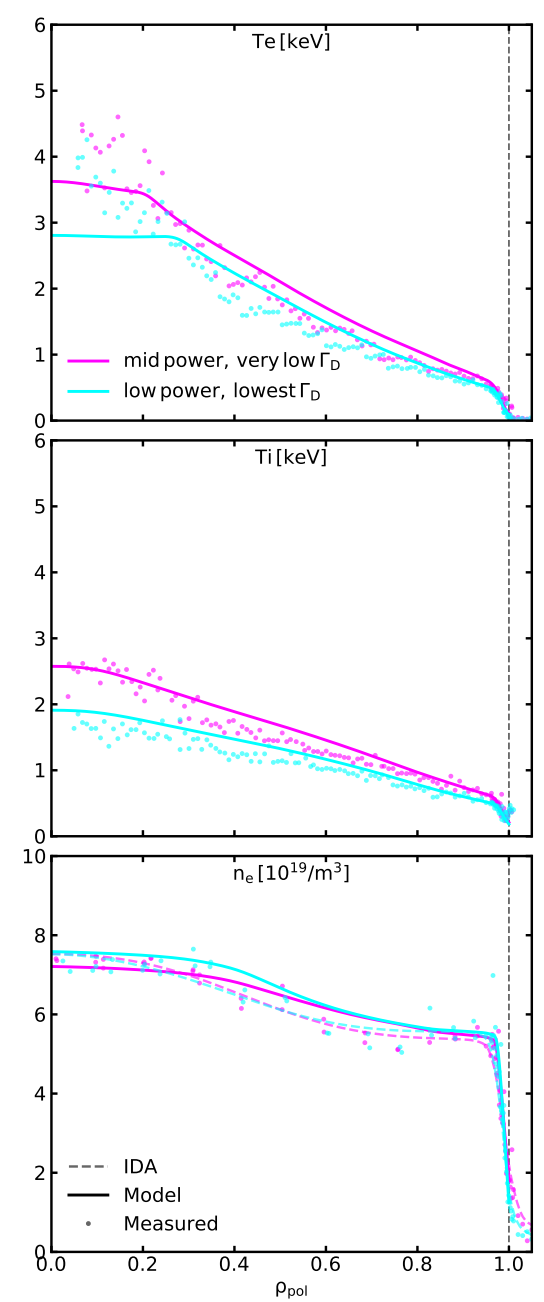

Figure 15. Experimental measurements (dots), and predicted profiles (lines) of electron and ion temperature, and electron density for the different levels of heating power and fueling rate. The dashed lines show the electron density profile obtained with the Integrated Data Analysis (IDA).

$\Gamma_{\mathrm{D}} \sim 0.15\left[10^{22} \mathrm{e} / \mathrm{s}\right]$, so most of the particle source is given by recycling. In the time window with higher heating power the fueling rate is larger by more than $100 \%$ to satisfy the requirements on the density, meaning that the pedestal particle transport has increased. The separatrix density has also increased due to the higher divertor neutral pressure (caused by higher fueling and NBI power), so the decay of the neutral density in the SOL is stronger, but we found that this effect is not strong enough to explain alone the higher fueling requirement: with the same value of pedestal particle diffusion coefficient for the 2 different cases, the change in the source of neutrals is not large enough to reproduce the change in the pedestal density gradient.

Figure 16 shows how the pedestal electron heat diffusivity $\chi_{\mathrm{e}, \text { ped }}$ increases with increased heating power, causing an increase in the particle diffusion coefficient (we 
Integrated modeling of ASDEX Upgrade plasmas combining core, pedestal and scrape-off layer physics26
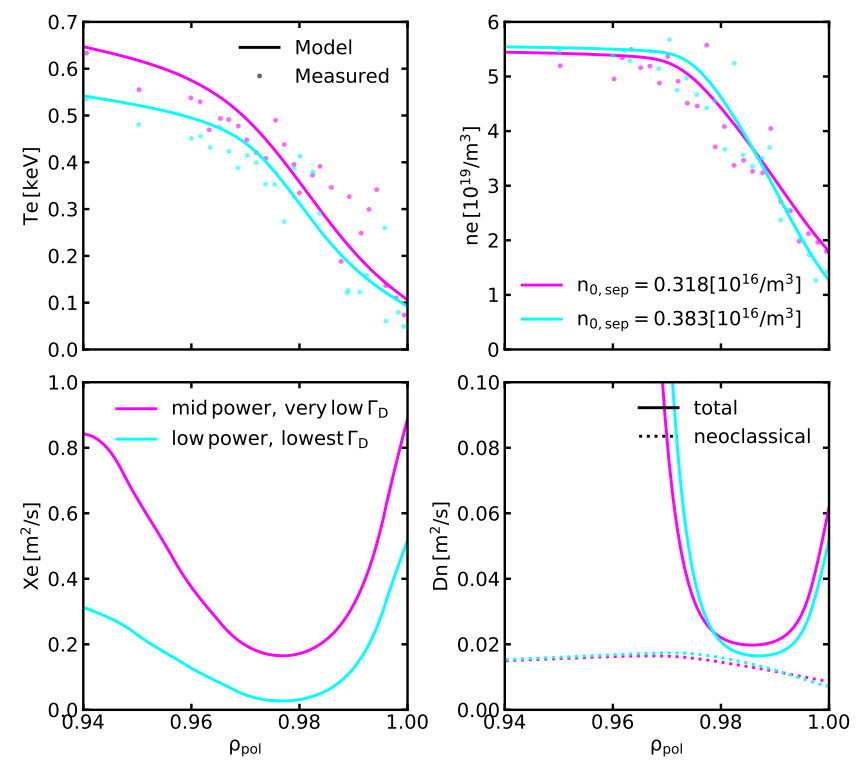

Figure 16. Predicted profiles (solid lines) of the pedestal electron heat diffusivity (lower left), and particle diffusivity (lower right) with its neoclassical component (dashed) for the different levels of heating power and fueling rate, and resulting electron temperature (upper left) and density (upper right) profiles. The measurements are represented by the dots. The label in the upper right figure shows the values of the neutral density at the separatrix for the two cases.

recall that $\left.D_{\mathrm{n}, \text { ped }}=c_{\mathrm{D} / \chi} \chi_{\mathrm{e} \text {,ped }}+D_{\mathrm{n}, \text { neo }}\right)$, and therefore causing a reduction in the pedestal density gradient. The relative increase of total heating power produced by an increase of the NBI heating is significantly larger than the corresponding relative increase of the total particle flux in the pedestal, which is dominated by the neutrals coming from the wall.

The elements included in the model, that describe all these effects, especially the pedestal particle transport that we find to increase with increasing heating power, can predict with excellent agreement the pedestal density profile. In particular we find that the pedestal density gradient decreases with increasing heating power, due to higher pedestal transport, and this is also observed from the measurements and described by the model for the power scan case at fixed fueling rate, as can be seen in figure 17 .

Figure 18 shows the ratio between the electron density at the pedestal top and at the separatrix $n_{\mathrm{e}, \mathrm{top}} / n_{\mathrm{e}, \mathrm{sep}}$, which is a proxy for the pedestal logarithmic density gradient, for the three power scans at the different fueling levels (color bar). As one can see, the prediction of the model (diamonds) reproduces the trend of the measurements (crosses), and most importantly one can see that with constant fueling rate the increasing heating power cause a reduction in the pedestal logarithmic density gradient. We associate this to an increase of the pedestal particle transport, caused by the increased hating power. This is also in line with Ref. [8, 72, 73], where it is observed that $n_{\mathrm{e}, \text { top }}$ has a negative dependence on NBI power, and is a step forward respect to the work on the integrated model in Ref. [29] where it is reported that a 
Integrated modeling of ASDEX Upgrade plasmas combining core, pedestal and scrape-off layer physics 27
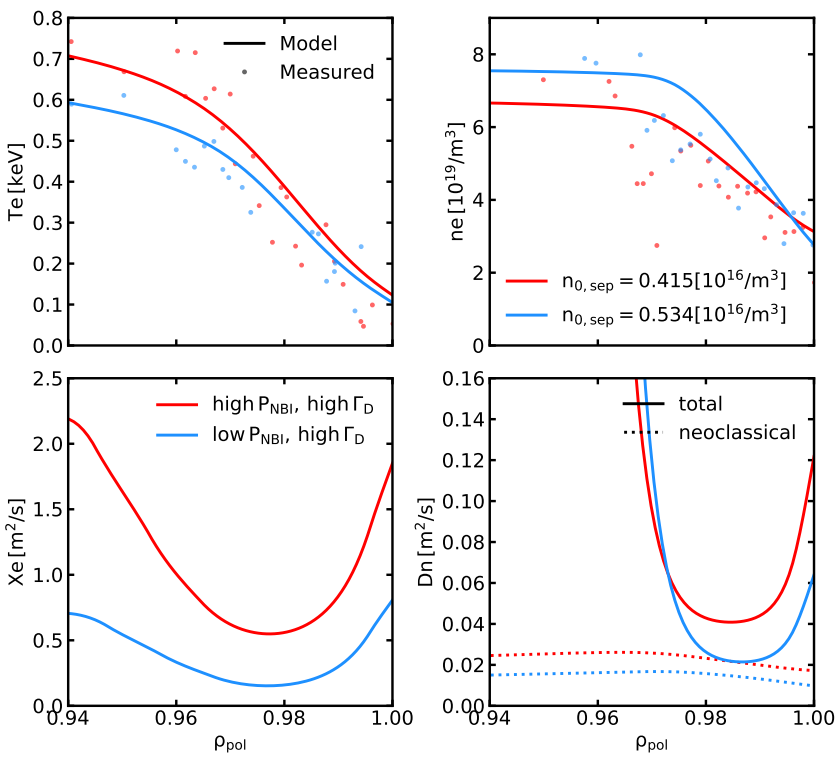

Figure 17. Predicted profiles (solid lines) of the pedestal electron heat diffusivity (lower left), and particle diffusivity (lower right) with its neoclassical component (dashed) for the different levels of heating power, and resulting electron temperature (upper left) and density (upper right) profiles. The measurements are represented by the dots. The label in the upper right figure shows the values of the neutral density at the separatrix for the two cases.

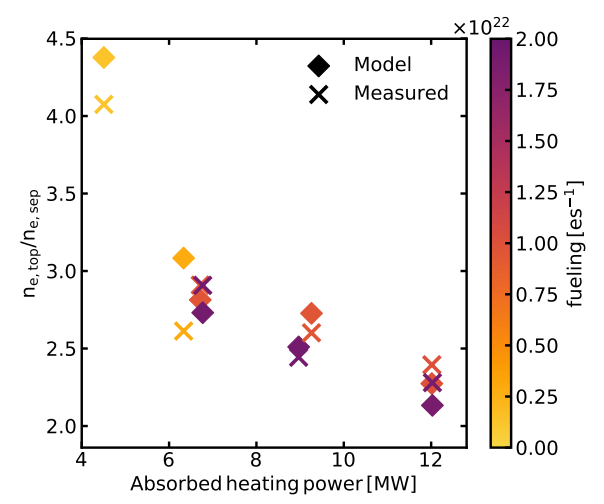

Figure 18. Dependence of the ratio between the pedestal top density and the separatrix density on the heating power, for different levels of fueling.

limitation of the neutral penetration model used to predict the pedestal density is the fact that it could not capture the power dependence on the pedestal density due to missing transport physics. 
Integrated modeling of ASDEX Upgrade plasmas combining core, pedestal and scrape-off layer physics 28

\subsection{Current scan}

Combining the discharge \#34153 with the stationary phase at lowest fueling rate from discharge \#33173 we obtain a scan in plasma current $I_{\mathrm{p}}=0.6-1 \mathrm{MA}$ keeping constant all the remaining parameters, with constant fueling rate instead of constant line averaged density.
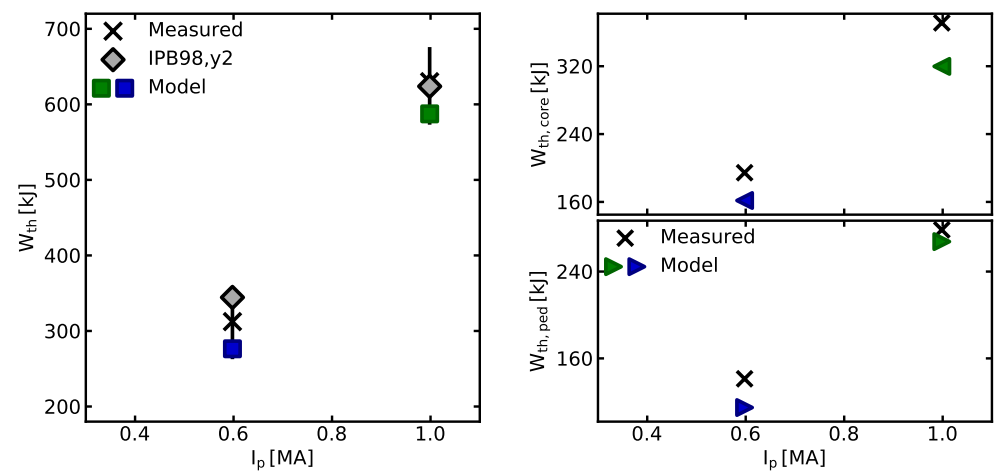

Figure 19. Thermal energy predicted by the model (squares), by the IPB98 $(\mathrm{y}, 2)$ (diamonds), and measured (black crosses) as a function of the plasma current (left). Experimental and predicted thermal energy separated in the pedestal and core components (right).

In figure 19 we can see that the change in the thermal energy caused by the different values of plasma current is well captured by the model, and the accuracy of its prediction is comparable to that of the IPB98 $(\mathrm{y}, 2)$. The case at $I_{\mathrm{p}}=1[\mathrm{MA}]$ has been already discussed in the fueling scan section. The predicted pedestal for the case at $I_{\mathrm{p}}=0.6[\mathrm{MA}]$ has a slightly lower top pressure with respect to the measurements, probably due to the fact that TGLF predicts a very large electron heat flux at the pedestal top $\left(\rho_{\text {tor }} \sim 0.78\right)$, affecting also the electron heat transport coefficients in the inner part of the pedestal, and resulting in a lower pedestal top electron temperature, as can be seen in figure 20. It is also interesting to notice that the increase of the density with $I_{\mathrm{p}}$ is well captured. The model can reproduce this experimental feature because the value of $\chi_{\mathrm{e} \text {,ped }}$, resulting from imposing $\left\langle\nabla T_{\mathrm{e}}\right\rangle / T_{\mathrm{e} \text {,ped }}=0.5$, decreases with increasing $I_{\mathrm{p}}$, causing a reduction in $D_{\mathrm{n}, \text { ped }}$, and therefore causing an increase in the pedestal density gradient. Overall, the TGLF prediction describes well the core kinetic profiles for this case, although in the inner part of the plasma the density gradient is underestimated. As future work, we will extend the validation of TGLF on more cases with a similar value of $q_{95}$.

\section{Summary and conclusions}

A new integrated modeling approach that describes the entire confined plasma domain, including the pedestal, up to the last closed flux surface, allows us to accurately predict plasma confinement only using global parameters as inputs. This was achieved by including into the ASTRA transport code a new pedestal transport model, which allows for a simultaneous evolution of the core and pedestal kinetic profiles, and a simple SOL model, which sets the boundary conditions. 
Integrated modeling of ASDEX Upgrade plasmas combining core, pedestal and scrape-off layer physics29
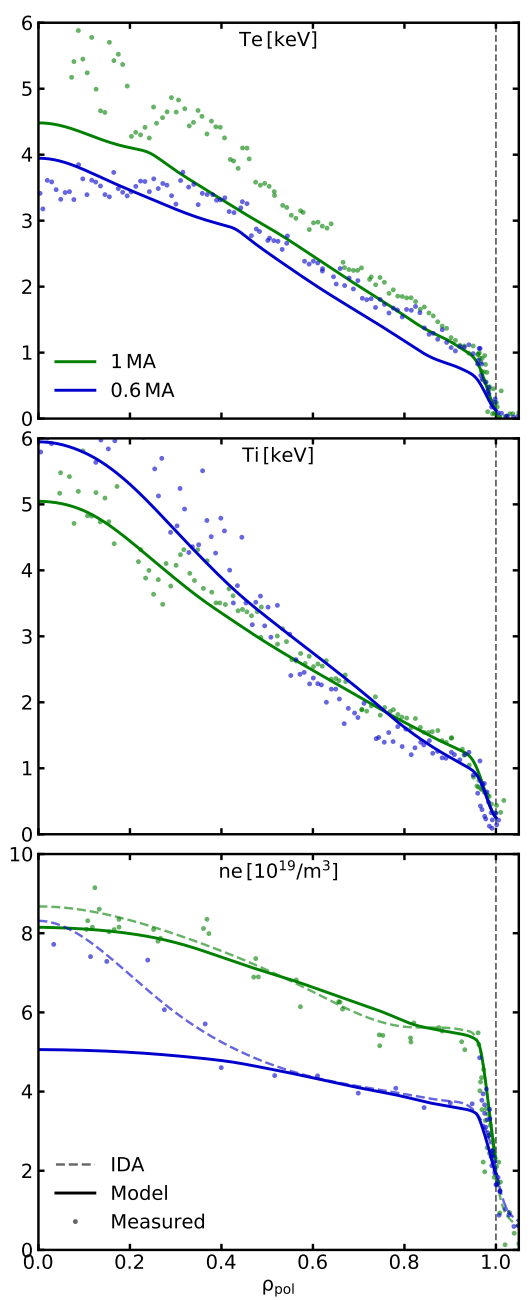

Figure 20. Experimental measurements (dots), and predicted profiles (lines) of electron and ion temperature, and electron density for the different levels of the plasma current. The dashed lines show the electron density profile obtained with the Integrated Data Analysis (IDA).

The core transport coefficients are given by the TGLF model, which is interfaced with the pedestal transport model using a radial buffer in the transition between the TGLF boundary and the pedestal top $\left(0.78<\rho_{\text {tor }}<\rho_{\text {ped }}^{\text {top }}\right)$ where we include an additional value to the heat and particle transport coefficients $\left(\chi_{\mathrm{e}, \mathrm{tr}}, \chi_{\mathrm{i}, \mathrm{tr}}, D_{\mathrm{n}, \mathrm{tr}}\right)$ in order to obtain smooth gradients of the kinetic profiles. These additional values are composed of two terms: one that is constant and one that is proportional to the pedestal electron heat diffusivity $\chi_{\mathrm{tr}}=c_{1}+c_{2} \chi_{\text {ped }}$, where $c_{1}$ and $c_{2}$ are assumed constants, and are different for electrons and ions. This approach was necessary as TGLF would otherwise (at least in the analyzed cases) highly over-predict the electron heat fluxes while strongly under-predicting particle and ion heat fluxes. In the region just inside the pedestal top, this results in an under prediction of 
the electron temperature peaking and in a over prediction of the density and ion temperature peaking. The reasons of this mismatch between the experiment and the TGLF prediction seems to be related to an underestimation of ITGs and a consequent overestimation of the ETGs by TGLF, as suggested by the analysis of the heat flux spectra. In these conditions, this leads to large electron heat fluxes, and correspondingly low ion heat fluxes in the TGLF prediction.

The pedestal transport model is based on the experimental observation $<\nabla T_{\mathrm{e}}>$ $/ T_{\mathrm{e}, \mathrm{ped}} \approx$ const $=0.5$ in a multi-device analysis [40], and gives a new transport constraint for the pedestal evolution in the inter-ELM recovery phase, which is different from the usual technique adopted by the EPED model $\left(\Delta_{\psi \mathrm{N}} \propto \sqrt{\beta_{\mathrm{p}, \mathrm{ped}}}\right)$.

The SOL model consists of a set of analytical formulas which calculate $n_{\mathrm{e}, \mathrm{sep}}$, $T_{\mathrm{e}, \mathrm{sep}}, T_{\mathrm{i}, \mathrm{sep}}$, and the source of neutrals at the separatrix, and has proven to robustly describe the effect of the fueling rate on $n_{\mathrm{e}, \mathrm{sep}}$ for the AUG cases considered. We have shown that a self-consistent treatment of the boundary conditions is a key element of this approach, and is necessary to capture the dependence of the pedestal pressure on the separatrix density, and therefore on the fueling rate, increasing the detail of the physics describing pedestal and global confinement. We have also shown that the new pedestal transport model gives an accurate estimation of the pedestal structure, bringing for the first time (to our knowledge) the capability of predicting separately the pedestal profiles of the electron temperature, and the ion temperature.

This new approach also increases the predictive capabilities of previous integrated models by simulating the pedestal density profile, and capturing its dependence on the gas-puff and heating power. The fact that the model is more accurate with respect to the scaling laws and captures the effect of fueling correctly is a very important aspect for the study of the scenarios for ITER and future fusion reactors, since the fusion gain is proportional to the $\mathrm{H}$-factor with the power of 3 [74], and in this case the $\mathrm{H}_{98(\mathrm{y}, 2)}$ error is up to $25 \%$, which means an error on the fusion gain $>50 \%$. Power exhaust sets constraints on the possible variation of the fueling rate, therefore it is important to take into account the effect that the operating conditions have on the simulations used to estimate the fusion performance.

Overall, the capability of simulating the kinetic profiles of the confined plasma increases the accuracy and the reliability in the prediction of energy confinement with respect to $0 \mathrm{D}$ scaling laws, as a $1 \mathrm{D}$ model can include the description of the physics phenomena which are strongly dependent on the gradients of the kinetic quantities, both in the core and in the pedestal regions. In particular we have shown that the density profile affects the shapes of the pressure gradients, which has a strong impact on the pedestal stability.

Of course the validity of the model on more experimental cases needs to be tested, therefore we are planning to extend this application to a larger number of AUG discharges. The extension of this test to other tokamaks is also considered for future work. Another part of future work will be the inclusion of the modeling of the toroidal rotation, which could be performed by fixing the boundary condition at the pedestal top, as done in Ref. [75]. The model contains some heuristic elements and has been tested (and is therefore valid) for AUG, but we expect that they should be applicable also to other machines. In particular the pedestal model is based on experimental observations that we expect to be applicable to other devices since it relies on a multimachine analysis which has identified a common, although dimensional, parameter. Of course this pedestal transport model can be easily and readily replaced by a theorybased model in case this becomes available. The main aspects which create a strong machine dependency are the geometry of the divertor and its baffles, and the location 
of the gas valves, which would probably make the estimation of the divertor neutral pressure $p_{0}$ not valid for different machines or divertors. A new scaling should be then derived for the tokamak of interest, using either experimental measurements if available, and/or synthetic data from simulations, particularly for non existing devices.

\section{Acknlowledgments}

The Authors are grateful to A. Ho for providing the GPR fitting tool, to R. Fischer for assistance in AUG data analysis, and finally to H. Zohm for fruitful discussions regarding the modeling of the pedestal.

This work has been carried out within the framework of the EUROfusion Consortium and has received funding from the Euratom research and training programme 20142018 and 2019-2020 under grant agreement number 633053. The views and opinions expressed herein do not necessarily reflect those of the European Commission.

\section{References}

[1] H. Zohm et al. "On the physics guidelines for a tokamak DEMO". In: Nuclear Fusion 53.7 (2013), p. 073019.

[2] Hartmut Zohm. "On the size of tokamak fusion power plants". In: Philosophical Transactions of the Royal Society A: Mathematical, Physical and Engineering Sciences 377.2141 (2019), p. 20170437.

[3] Y. Sarazin et al. "Impact of scaling laws on tokamak reactor dimensioning". In: Nuclear Fusion 60.1 (2019), p. 016010.

[4] S.M Kaye et al. "ITER L mode confinement database". In: Nuclear Fusion 37.9 (1997), pp. 1303-1328.

[5] ITER Physics Basis Editors. In: Nucl. Fusion 39 (1999), p. 2175.

[6] D.C McDonald et al. "Recent progress on the development and analysis of the ITPA global H-mode confinement database". In: Nuclear Fusion 47.3 (2007), pp. $147-174$.

[7] T C Luce, C C Petty, and J G Cordey. "Application of dimensionless parameter scaling techniques to the design and interpretation of magnetic fusion experiments". In: Plasma Physics and Controlled Fusion 50.4 (2008), p. 043001.

[8] C.D. Challis et al. "Improved confinement in JET high plasmas with an ITERlike wall". In: Nuclear Fusion 55.5 (2015), p. 053031.

[9] J Garcia and D Cambon and. "On the universality of power laws for tokamak plasma predictions". In: Plasma Physics and Controlled Fusion 60.2 (2018), p. 025028.

[10] J.E. Kinsey, G.M. Staebler, and R.E. Waltz. "The first transport code simulations using the trapped gyro-Landau-fluid model". In: Phys. Plasmas 15 (2008), p. 055908.

[11] J. Candy et al. "Tokamak profile prediction using direct gyrokinetic and neoclassical simulation". In: Phys. Plasmas 16 (2009), p. 060704.

[12] J.E. Kinsey et al. "ITER predictions using the GYRO verified and experimentally validated trapped gyro-Landau fluid transport model". In: Nucl. Fusion 51 (2011), p. 083001.

[13] M. Murakami et al. "Integrated modelling of steady-state scenarios and heating and current drive mixes for ITER". In: Nucl. Fusion 51.10 (2011), p. 103006. 
[14] L. Garzotti et al. "Simulations of density profiles, pellet fuelling and density control in ITER". In: Nuclear Fusion 52.1 (2011), p. 013002.

[15] G.L. Falchetto and et al. "The European Integrated Tokamak Modelling (ITM) effort: achievements and first physics results". In: Nucl. Fusion 54 (2014), p. 043018.

[16] M Romanelli et al. "JINTRAC: A system of codes for integrated simulation of tokamak scenarios". In: Plasma Fusion Res (2014).

[17] S. Breton et al. "First principle integrated modeling of multi-channel transport including Tungsten in JET". In: Nuclear Fusion 58.9 (2018), p. 096003.

[18] O. Linder et al. "Flux-driven integrated modelling of main ion pressure and trace tungsten transport in ASDEX Upgrade". In: Nuclear Fusion 59.1 (2018), p. 016003 .

[19] E. Fable et al. "The role of the source versus the collisionality in predicting a reactor density profile as observed on ASDEX Upgrade discharges". In: Nuclear Fusion 59.7 (2019), p. 076042.

[20] Glenn Bateman, Thawatchai Onjun, and Arnold H Kritz. "Integrated predictive modelling simulations of burning plasma experiment designs". In: Plasma Physics and Controlled Fusion 45.11 (2003), p. 1939.

[21] R.V. Budny et al. "Predictions of H-mode performance in ITER". In: Nucl. Fusion 48 (2008), p. 075005.

[22] R.V. Budny. "Comparisons of predicted plasma performance in ITER H-mode plasmas with various mixes of external heating". In: Nucl. Fusion 49.8 (2009), p. 085008 .

[23] A.H. Kritz et al. "Integrated modelling for prediction of optimized ITER performance". In: Nucl. Fusion 51.12 (2011), p. 123009.

[24] T. Rafiq et al. "Fusion power production in ITER baseline H-mode scenarios". In: Phys. Plasmas 22.4, 042511 (2015).

[25] M Murakami et al. "Exploration of ITER Steady-State Scenarios Using FASTRAN/IPS Integrated Transport Modeling". In: APS Meeting Abstracts. Vol. 1. 2013, 8021P.

[26] FM Poli et al. "External heating and current drive source requirements towards steady-state operation in ITER". In: Nucl. Fusion 54.7 (2014), p. 073007.

[27] O. Meneghini et al. "Integrated fusion simulation with self-consistent corepedestal coupling". In: Physics of Plasmas 23.4, 042507 (2016).

[28] O. Meneghini et al. "Self-consistent core-pedestal transport simulations with neural network accelerated models". In: Nuclear Fusion 57.8 (2017), p. 086034.

[29] S Saarelma et al. "Integrated modelling of H-mode pedestal and confinement in JET-ILW". In: Plasma Physics and Controlled Fusion 60.1 (2017), p. 014042.

[30] G M Staebler, J E Kinsey, and R E Waltz. "A theory-based transport model with comprehensive physics". In: Phys. Plasmas 14 (2007), p. 055909.

[31] G.M. Staebler et al. "A model of the saturation of coupled electron and ion scale gyrokinetic turbulence". In: Nuclear Fusion 57.6 (2017), p. 066046.

[32] C. Bourdelle et al. "A new gyrokinetic quasilinear transport model applied to particle transport in tokamak plasmas". In: Physics of Plasmas 14.11, 112501 (2007). 
[33] J Citrin et al. "Tractable flux-driven temperature, density, and rotation profile evolution with the quasilinear gyrokinetic transport model QuaLiKiz". In: Plasma Physics and Controlled Fusion 59.12 (2017), p. 124005.

[34] Summary of the ITER Final Design Report. ITER EDA Documentation Series 22. Vienna: INTERNATIONAL ATOMIC ENERGY AGENCY, 2001.

[35] G V Pereverzev and P N Yushmanov. "ASTRA-an automatic system for transport analysis in a tokamak". In: IPP-report (1991).

[36] W.A. Houlberg et al. "Bootstrap current and neoclassical transport in tokamaks of arbitrary collisionality and aspect ratio". In: Phys. Plasmas 4 (1997), p. 3230.

[37] A.B. Mihailovskii. "inot availablę". In: Plasma Phys. Rep. 23 (1997), p. 844.

[38] Stangeby. The Plasma Boundary of Magnetic Fusion Devices. Ed. by IOP. IOP Publishing Ltd., 2000.

[39] P C Stangeby. "Basic physical processes and reduced models for plasma detachment". In: Plasma Physics and Controlled Fusion 60.4 (2018), p. 044022.

[40] PA Schneider et al. "Analysis of temperature and density pedestal gradients in AUG, DIII-D and JET". In: Nuclear Fusion 53.7 (2013), p. 073039.

[41] E Poli, AG Peeters, and GV Pereverzev. "TORBEAM, a beam tracing code for electron-cyclotron waves in tokamak plasmas". In: Computer physics communications 136.1 (2001), pp. 90-104.

[42] AA Ivanov et al. "New adaptive grid plasma evolution code SPIDER". In: 32nd EPS Conf. on Plasma Physics 29C (2005).

[43] A Kallenbach et al. "Neutral pressure and separatrix density related models for seed impurity divertor radiation in ASDEX Upgrade". In: Nuclear Materials and Energy 18 (2019), pp. 166-174.

[44] M Siccinio et al. "A 0D stationary model for the evaluation of the degree of detachment on the divertor plates". In: Plasma Physics and Controlled Fusion 58.12 (2016), p. 125011.

[45] A.W. Leonard et al. "Scaling radiative divertor solutions to high power in DIIID". In: Nuclear Fusion 52.6 (2012), p. 063015.

[46] T. Eich et al. "Inter-ELM Power Decay Length for JET and ASDEX Upgrade: Measurement and Comparison with Heuristic Drift-Based Model". In: Phys. Rev. Lett. 107 (21 2011), p. 215001.

[47] T. Eich et al. "Scaling of the tokamak near the scrape-off layer H-mode power width and implications for ITER". In: Nuclear Fusion 53.9 (2013), p. 093031.

[48] D. Brida et al. "Heat flux pattern in detached L-modes and ELM mitigated Hmodes with rotating magnetic perturbations in ASDEX Upgrade". In: Nuclear Fusion 57.11 (2017), p. 116006.

[49] A Kallenbach et al. "Parameter dependences of the separatrix density in nitrogen seeded ASDEX Upgrade H-mode discharges". In: Plasma Physics and Controlled Fusion 60.4 (2018), p. 045006.

[50] A. Herrmann et al. "Solid tungsten Divertor-III for ASDEX Upgrade and contributions to ITER". In: Nuclear Fusion 55.6 (2015), p. 063015.

[51] S. Potzel et al. "A new experimental classification of divertor detachment in ASDEX Upgrade". In: Nuclear Fusion 54.1 (2013), p. 013001. 
[52] S. Potzel et al. "Formation of the high density front in the inner far SOL at ASDEX Upgrade and JET". In: Journal of Nuclear Materials 463 (2015), pp. 541 -545 .

[53] A Kallenbach et al. "Analytical calculations for impurity seeded partially detached divertor conditions". In: Plasma Physics and Controlled Fusion 58.4 (2016), p. 045013.

[54] J Stober and the ASDEX Upgrade Team. "Improvement of ion-temperatureprofile determination from charge exchange measurements by inclusion of totalneutral-flux data". In: Plasma Physics and Controlled Fusion 39.7 (1997), pp. $1145-1152$.

[55] M. Willensdorfer et al. "Particle transport analysis of the density build-up after the L-H transition in ASDEX Upgrade". In: Nuclear Fusion 53.9 (2013), p. 093020.

[56] P.B. Snyder et al. "Development and validation of a predictive model for the pedestal height". In: Phys. Plasmas 16 (2009), p. 056118.

[57] PA Schneider et al. "Differences in the H-mode pedestal width of temperature and density". In: Plasma Physics and Controlled Fusion 54.10 (2012), p. 105009.

[58] D.R. Hatch et al. "Microtearing turbulence limiting the JET-ILW pedestal". In: Nuclear Fusion 56.10 (2016), p. 104003.

[59] D.R. Hatch et al. "Gyrokinetic study of ASDEX Upgrade inter-ELM pedestal profile evolution". In: Nuclear Fusion 55.6 (2015), p. 063028.

[60] M. Kotschenreuther et al. "Gyrokinetic analysis and simulation of pedestals to identify the culprits for energy losses using "fingerprints"'. In: Nuclear Fusion 59.9 (2019), p. 096001.

[61] F.Porcelli, D.Boucher, and M.N.Rosenbluth. "Model for the sawtooth period and amplitude". In: Plasma Phys. Control. Fusion 38 (12 1996).

[62] F. Sommer et al. "Transport properties of H-mode plasmas with dominant electron heating in comparison to dominant ion heating at ASDEX Upgrade". In: Nuclear Fusion 55.3 (2015), p. 033006.

[63] E Fable et al. "Novel free-boundary equilibrium and transport solver with theory-based models and its validation against ASDEX Upgrade current ramp scenarios". In: Plasma Phys. Control. Fusion 55.12 (2013), p. 124028.

[64] I. Erofeev et al. "Theory-based modeling of LOC-SOC transitions in ASDEX Upgrade". In: Nucl. Fusion 57.12 (2017), p. 126067.

[65] C Konz et al. "First physics applications of the Integrated Tokamak Modelling (ITM-TF) tools to the MHD stability analysis of experimental data and ITER scenarios". In: EPS (2011), O2.

[66] R Fischer. "Effective ion charge $Z_{\text {eff }}$ from integrated analysis of multiple diagnostics at ASDEX Upgrade". In: 37th EPS Conf. on Plasma Physics 34A (2010).

[67] R. Fischer et al. "Integrated Data Analysis of Profile Diagnostics at ASDEX Upgrade". In: Fusion Science and Technology 58.2 (2010), pp. 675-684.

[68] A. Ho et al. "Application of Gaussian process regression to plasma turbulent transport model validation via integrated modelling". In: Nuclear Fusion 59.5 (2019), p. 056007. 
[69] M G Dunne et al. "The role of the density profile in the ASDEX-Upgrade pedestal structure". In: Plasma Physics and Controlled Fusion 59.1 (2016), p. 014017.

[70] E. Stefanikova et al. "Effect of the relative shift between the electron density and temperature pedestal position on the pedestal stability in JET-ILW and comparison with JET-C". In: Nuclear Fusion 58.5 (2018), p. 056010.

[71] L. Frassinetti et al. "Role of the pedestal position on the pedestal performance in AUG, JET-ILW and TCV and implications for ITER". In: Nuclear Fusion 59.7 (2019), p. 076038.

[72] H. Urano et al. "Characterization of electron density based on operational parameters in JET H-mode plasmas with C and ILW". In: Proc. 43rd EPS Conference on Plasma Physics. 2016.

[73] C.F. Maggi et al. "Pedestal confinement and stability in JET-ILW ELMy Hmodes". In: Nuclear Fusion 55.11 (2015), p. 113031.

[74] A.G. Peeters, C. Angioni, and A.C.C. Sips. "On the extrapolation to ITER of discharges in present tokamaks". In: Nuclear Fusion 47.9 (2007), pp. 1341-1345.

[75] J. M. Park et al. "Integrated modeling of high N steady state scenario on DIIID". In: Physics of Plasmas 25.1 (2018), p. 012506. eprint: https://doi.org/ 10.1063/1.5013021. 